\title{
USE OF GROUND BASED SIGNALS OF OPPORTUNITY FOR SMART PROJECTILE NAVIGATION
}

\author{
A Thesis \\ Presented to \\ The Academic Faculty
}

By

James E. Wright

\author{
In Partial Fulfillment \\ Of the Requirements for the Degree \\ Master of Science in Aerospace Engineering
}

Georgia Institute of Technology

May 2010

Copyright (C) James E. Wright 2010 


\section{USE OF GROUND BASED SIGNALS OF OPPORTUNITY FOR SMART PROJECTILE NAVIGATION}

Approved by:

Dr. Mark Costello, Advisor School of Aerospace Engineering Georgia Institute of Technology

Dr. John-Paul Clarke School of Aerospace Engineering Georgia Institute of Technology

Dr. Gene Cooper Weapons and Materials Research Directorate Army Research Lab

Date Approved: December 3, 2009 


\section{ACKNOWLEDGMENTS}

Thank you to Dr. Mark Costello for his guidance over the past two years. I appreciate your encouragement to study a topic related to alternative navigation solutions and your efforts in providing a wealth of resources to accomplish the task.

I also would like to thank Dr. J.P. Clarke and Dr. Gene Cooper for serving on my thesis committee and offering a great deal of advice and assistance.

Finally, I would like to acknowledge the graduate students, and other individuals from the Army Research Lab for their help and support: Dr. Jonathan Rogers, Dr. Frank Fresconi, Dr. Mark Ilg, Dave Lyon, Peter Plostins and James Maley. 


\section{TABLE OF CONTENTS}

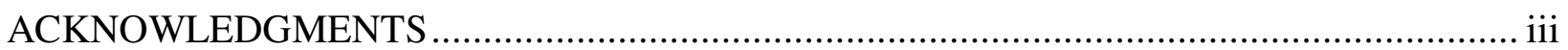

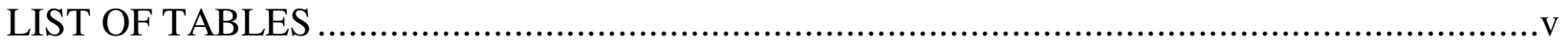

LIST OF FIGURES ...................................................................................... vi

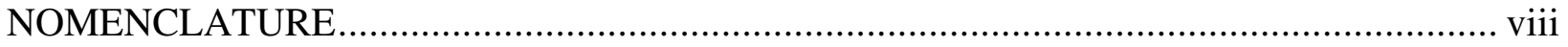

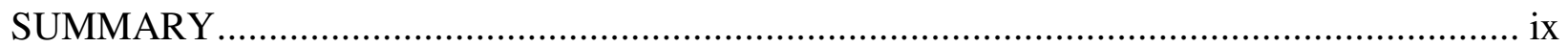

CHAPTER 1 - INTRODUCTION .........................................................................

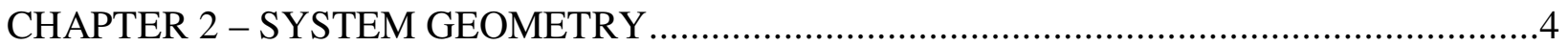

CHAPTER 3 - NAVIGATION SOLUTION ...........................................................6

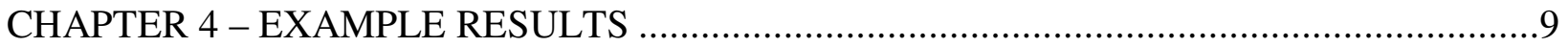

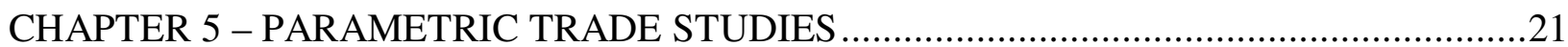

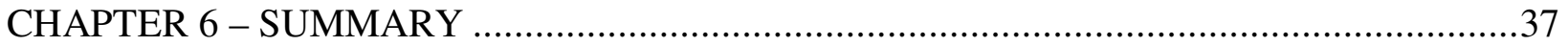

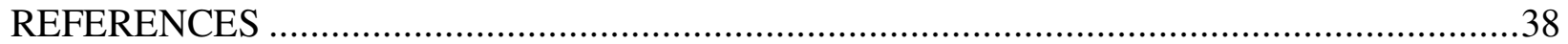




\section{LIST OF TABLES}

Table 1. Error Characteristics for Range and Range Rate ................................................6

Table 2. 95\% Confidence Spheres vs. Location of Signal Sources for 3s, 15s and 30s .............31

Table 3. 95\% Confidence Spheres vs. Scenarios for 3s, 15s and 30s.................................36 


\section{LIST OF FIGURES}

Figure 1. System Geometry for a Single Signal Source........................................................

Figure 2. Line of Sight for Several Signal Sources ...........................................................

Figure 3. Location of Signal Sources - Baseline Configuration ............................................10

Figure 4. Range vs. Time - Baseline Configuration...............................................................1

Figure 5. Cross Range vs. Time - Baseline Configuration .......................................................12

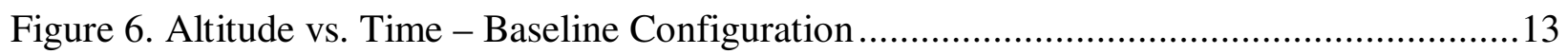

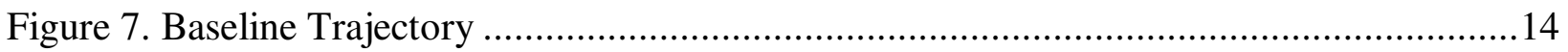

Figure 8. Position Residuals vs. Time - Baseline Configuration ..............................................15

Figure 9. Velocity Residuals vs. Time - Baseline Configuration ..............................................16

Figure 10. 3D Trajectory with Elevation Data and Areas of Focus: 3s, 15s and 30s ..................17

Figure 11. Histograms of X Calculated at $t=3 \mathrm{~s}, 15 \mathrm{~s}$ and 30s - Monte Carlo Simulation ...........18

Figure 12. Histograms of $\mathrm{Y}$ Calculated at $\mathrm{t}=3 \mathrm{~s}, 15 \mathrm{~s}$ and $30 \mathrm{~s}-$ Monte Carlo Simulation ...........19

Figure 13. Histograms of $Z$ Calculated at $t=3 \mathrm{~s}, 15 \mathrm{~s}$ and $30 \mathrm{~s}-$ Monte Carlo Simulation ...........20

Figure 14. NED Dataset for Bonneville Salt Flats (Flat Landscape)........................................22

Figure 15. NED Dataset for Colorado Mountains (Mountainous Landscape) ..............................23

Figure 16. NED Dataset for Atlanta (Urban Landscape) .........................................................24

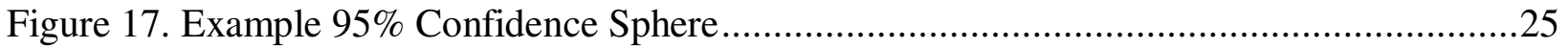

Figure 18. 95\% Confidence Position Spheres vs. Grid Size for 3s, 15s and 30s ........................27

Figure 19. 95\% Confidence Velocity Spheres vs. Grid Size for 3s, 15s and 30s.......................28

Figure 20. 95\% Confidence Position Spheres vs. Number of Signal Sources for 3s, 15s and 30s29 
Figure 21. 95\% Confidence Velocity Spheres vs. Number of Signal Sources for 3s, 15s and 30s

Figure 22. 95\% Confidence Position Spheres vs. Size of Error Level for 3s, 15 s and 30s ..........32

Figure 23. 95\% Confidence Velocity Spheres vs. Size of Error Level for 3s, 15s and 30s.........33

Figure 24. 95\% Confidence Position Spheres vs. Error in Signal Source Position for 3s, 15s and $30 \mathrm{~s}$

Figure 25. 95\% Confidence Velocity Spheres vs. Error in Signal Source Position for 3s, 15s and 30s. 


\section{NOMENCLATURE}

$b_{i}:$ Gaussian bias term

$E_{k}:$ Error vector

$\bar{I}_{I}, \bar{J}_{I}, \bar{K}_{I}:$ Inertial reference frame unit vectors

$J_{k}:$ Jacobian of kth iteration

$P_{k}$ : Vector of kth unknown parameters

$\bar{r}_{o \rightarrow p}$ : Distance vector from origin of the inertial reference frame to mass center of projectile

$\bar{r}_{o \rightarrow s_{i}}$ : Distance vector from origin of the inertial reference frame to ith signal source

$R_{i}$ : Range from ith signal source to projectile

$\tilde{R}_{i}$ : Pseudorange from ith signal source to projectile

$\dot{R}_{i}$ : Range rate from ith signal source to projectile

$\dot{\tilde{R}}_{i}$ : Pseudorange rate from ith signal source to projectile

$\bar{v}_{P / I}$ : Translational velocity of the mass center of projectile in the inertial reference frame

$\bar{v}_{S_{i} / I}$ : Translational velocity of ith signal source in the inertial reference frame

$w_{i}$ : Gaussian random number

$x, y, z$ : Position vector components of the composite body center of mass expressed in the inertial reference frame

$x_{s_{i}}, y_{s_{i}}, z_{s_{i}}$ : Position vector components of the ith signal source expressed in the inertial reference frame

$\dot{x}, \dot{y}, \dot{z}$ : Velocity vector components of the composite body center of mass expressed in the inertial reference frame

$\dot{x}_{s_{i}}, \dot{y}_{s_{i}}, \dot{z}_{s_{i}}$ : Velocity vector components of the ith signal source expressed in the inertial reference frame

$\alpha_{k}:$ Line search parameter

$\sigma_{i}:$ Error characteristic for signal 


\section{SUMMARY}

GPS is a widely accepted means of navigation, whether it is for civilian or military means. With the implementation of GPS on smart projectiles, these weapons have been able to achieve remarkable accuracy. Even though the improvements in accuracy are impressive, GPS signals are susceptible to jamming and spoofing by a sufficiently motivated enemy. The work reported here examines the viability of constructing a navigation solution using ground based signals of opportunity that provide range and range rate information. Using a generalized sensor model encompassing the key error terms, a variety of physical devices are included in the analysis.

For a typical indirect fire trajectory, navigation solutions are computed as a function of the number and density of signal sources, terrain type, and sensor errors. Systematic studies were performed using these parameters in order to better understand the merits and demerits of this type of system to create a useful navigation solution. Based on these studies, results indicate that navigation solutions can be computed with the same accuracy as current GPS systems with a moderate number of signal sources. Generally, more accurate solutions are

obtained when the projectile is directly over the signal sources and there is variation of signal source location in all three axes. 


\section{CHAPTER 1 - INTRODUCTION}

Global Positioning System (GPS) is more and more a ubiquitous part of society. For more than a decade, smart indirect fire projectiles and smart bombs have employed GPS position and velocity feedback to achieve remarkable accuracy. An early example is the Joint Direct Attack Munition (JDAM). By employing GPS, the JDAM achieved a circular error probable (CEP) of $10 \mathrm{~m}(\sim 33 \mathrm{ft})$, which was demonstrated during Operation Allied Force, the NATO bombing of Yugoslavia in $1999^{[1]}$. Another example is the Excalibur, a $155 \mathrm{~mm}$ extended range guided artillery shell. Unguided, it possesses a CEP of 200 to $300 \mathrm{~m}$ at moderate ranges, where the GPS guided derivative achieves a CEP of $10 \mathrm{~m}$, an astonishing increase in accuracy and precision ${ }^{[2]}$. While these improvements in accuracy are impressive, the improvement in accuracy is accompanied by a substantial increase in cost and sophistication of the weapon. Moreover, one of the more worrisome aspects of GPS enabled munitions is the fact that GPS signals can be easily jammed or spoofed by a sufficiently motivated enemy. A current method to mitigate GPS signal loss is to integrate an Inertial Navigation System (INS) along with a kalman filter to obtain a navigation solution. Also, when GPS signals are available, this system allows a navigation solution to be computed at higher rates than GPS alone can provide. For example, JDAM employs an INS to ensure that should GPS be lost, the munition can still hit its target. Solely relying on INS, the JDAM has a CEP of $30 \mathrm{~m}$, which is not as accurate as guidance by GPS, but still provides sufficient accuracy ${ }^{[1]}$.

A substantial amount of research has been performed on countering GPS jamming. A traditional approach to anti-jamming is to employ a six antenna element arranged in a hexagonal 
pattern around a central reference element, where all elements are connected to a central electronics box that controls the phase and gain of each element. By tuning each of the antennas independently a null can be placed in the direction of an undesired signal source. However, this concept is relatively large, $35 \mathrm{~cm}$, and only works with a few unwanted signal sources ${ }^{[3]}$. Two current anti-jamming methods are jammer signal power reduction or frequency adaptive processing. Jammer signal power reduction employs space-time adaptive processing, where each antenna array element is delayed using a set of tapped delay lines. Once again, the major issues with power reduction is the fact that there are a limited number of degrees of freedom for which this concept will work and the need for multiple antennas ${ }^{[3]}$. Frequency adaptive processing is an adaptive narrowband process and uses only a single antenna element, ideal for smart weapons. These systems attempt to minimize measured power based on the assumption that any measured power must be a jamming signal ${ }^{[4]}$. However, it is only effective against structured interference signals and not broadband interference. Additionally, smart weapons have begun to utilize an inertial navigation system (INS) as a method for navigating when GPS is jammed or denied. INSs utilize a set of accelerometers and gyroscopes to calculate certain quantities which can then be integrated to estimate the states of projectile, such as position and velocity. Highly accurate IMUs are expensive and can cost upwards of $\$ 100,000$. Lower cost and less accurate IMU's do find their way into smart weapons, but typically only as a backup should GPS signals be lost or jammed. Lower cost IMU's can be built-in house or purchased for around $\$ 3,000$ dollars ${ }^{[5]}$.

Another option for obtaining a navigation solution when GPS is not available is to use RF signals of opportunity (SOOP) produced from known sources. Similar to GPS navigation systems, this concept utilizes known positions of ground based signal sources and develops a pseudorange and pseudorange rate from the projectile to the source. By utilizing at least four 
sources, position and velocity can be computed. Savarese, Rabaey, and Beutel demonstrated this concept via multiple experiments, using AM signals. They provide examples and results of using SOOP to accurately map an entire network ${ }^{[6]}$. Similarly, robots are using active and passive

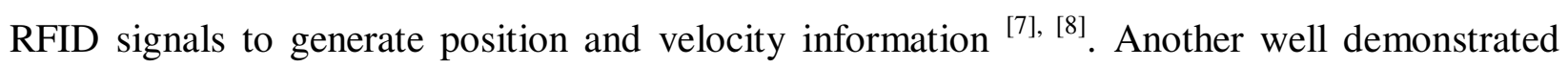
system employing this concept is eLORAN, which was recently chosen as the backup global navigation solution, should GPS be lost for an extended period of time. Currently eLORAN allows for $+/-8 \mathrm{~m}$ accuracy when roughly $165 \mathrm{~km}$ or less away from the transmitting towers ${ }^{[9]}$, [10], [11].

This paper explores the potential of creating a navigation solution for a smart projectile using an array of known ground based RF sources that emit pseudorange, and pseudorange rate information that can be received and processed by a smart projectile in real time. The main focus of the paper is exploring the effect of practical system features on the quality of the resulting navigation solution. The paper begins with a description of the system geometry considered along with the numerical algorithm employed for constructing the navigation solution, including documenting the error model for range and range rate information. The algorithm is subsequently used to predict a navigation solution of a smart indirect fire projectile, flying a typical trajectory. Parametric trade studies considering the number of active signal sources, location of signal sources, density of signal sources, terrain types and sensor error levels are conducted and contrasted with current GPS based navigation solutions. 


\section{CHAPTER 2 - SYSTEM GEOMETRY}

The scenario considered here consists of a projectile in atmospheric flight. The projectile is equipped with an electronics suite that processes measurements from $\mathrm{N}$ independent signal sources. For each signal source, the pseudorange and pseudorange rate from the projectile to the signal source is obtained at a given instant in time. The basic geometry is shown in Figure 1.

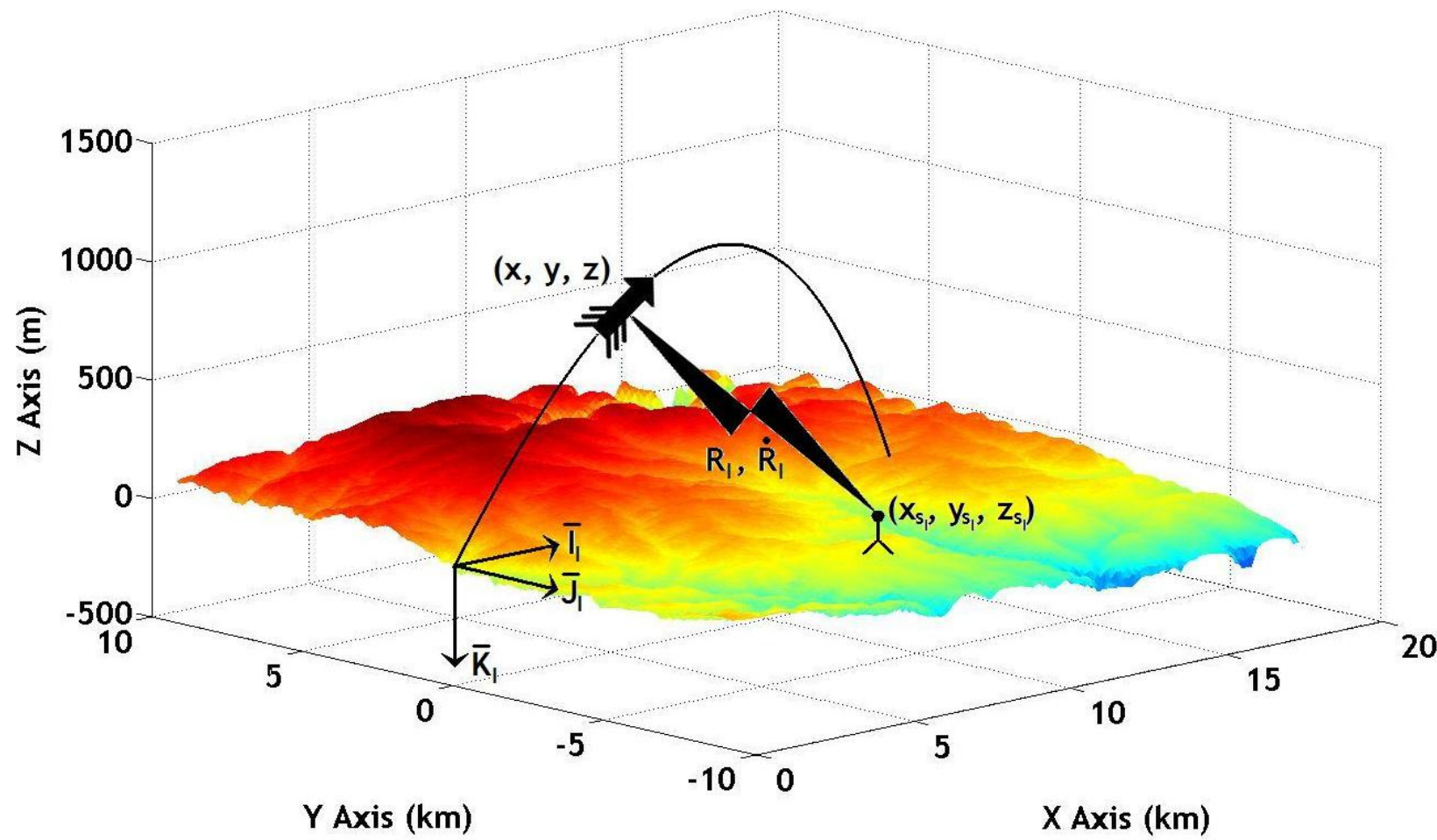

Figure 1. System Geometry for a Single Signal Source

The inertial reference frame is fixed to the surface of the earth and is arranged so that its origin is located close to the gun muzzle with the $\bar{I}_{I}$ axis pointing down range. The $\bar{J}_{I}$ axis points to the 
right when viewed from the rear and the $\bar{K}_{I}$ axis points into the ground. The location of each signal source is known in the inertial reference frame.

The distance vector from the origin of the inertial frame to the mass center of the projectile and the ith signal source is:

$$
\begin{gathered}
\bar{r}_{o \rightarrow p}=x \bar{I}_{I}+y \bar{J}_{I}+z \bar{K}_{I} \\
\bar{r}_{o \rightarrow s_{i}}=x_{s_{i}} \bar{I}_{I}+y_{s_{i}} \bar{J}_{I}+z_{s_{i}} \bar{K}_{I}
\end{gathered}
$$

while the corresponding translational velocities are:

$$
\begin{gathered}
\bar{v}_{P / I}=\dot{x} \bar{I}_{I}+\dot{y} \bar{J}_{I}+\dot{z} \bar{K}_{I} \\
\bar{v}_{S_{i} / I}=\dot{x}_{s_{i}} \bar{I}_{I}+\dot{y}_{s_{i}} \bar{J}_{I}+\dot{z}_{s_{i}} \bar{K}_{I}
\end{gathered}
$$

With these definitions, the range from the signal source to the associated projectile receiver is:

$$
\left|\bar{r}_{o \rightarrow s_{i}}-\bar{r}_{o \rightarrow p}\right|=R_{i}=\sqrt{\left(x-x_{s_{i}}\right)^{2}+\left(y-y_{s_{i}}\right)^{2}+\left(z-z_{s_{i}}\right)^{2}}
$$

And the range rate

$$
\dot{R}_{i}=\frac{\left(x-x_{s_{i}}\right)\left(\dot{x}-\dot{x}_{s_{i}}\right)+\left(y-y_{s_{i}}\right)\left(\dot{y}-\dot{y}_{s_{i}}\right)+\left(z-z_{s_{i}}\right)\left(\dot{z}-\dot{z}_{s_{i}}\right)}{R_{i}}
$$




\section{CHAPTER 3 - NAVIGATION SOLUTION}

The core signals used to create a navigation solution are the pseudorange and pseudorange rate from the signal source to the projectile receiver. Physically, this information can be extracted from an AM, television, cell phone tower, radio beacon or vision system. The first method for determining the necessary information uses received signal strength indication (RSSI), which employs the property that as a signal propagates forward the signal strength is reduced in a known manner, which in turn provides the distance between the signal source and receiver. The second method utilizes time-difference of arrival (TDOA) and two different receivers in order to calculate range information. It is assumed here that each signal is corrupted with gaussian noise and random bias in the form shown in Equation 7.

$$
\tilde{R}_{i}=R_{i}\left(1+\sigma_{i} w_{i}\right)+b_{i}
$$

Equation 7 is a generalized sensor model that permits modeling many different physical sensors by properly setting the error characteristics $\left(\sigma_{i}, b_{i}\right)$. Table 1 lists typical error values for several different signal sources.

Table 1. Error Characteristics for Range and Range Rate

\begin{tabular}{|ccc|}
\hline & $\sigma(\%)$ & $b(\mathrm{~m})$ \\
\hline eLORAN (90 - 110 kHz) & $0.00008-$ higher & $0.3-3.0$ \\
AM (520 - 1,710 kHz) & $0.0001-0.0007$ & $1.5-12.2$ \\
RFID (mHz) & $0.001-0.01$ & $0.2-0.5$ \\
\hline
\end{tabular}

[7], [8], [9], [10], [11], [13], [14] 
The basic navigation solution problem is to determine $x, y, z, \dot{x}, \dot{y}, \dot{z}$ given a set of $\mathrm{N}$

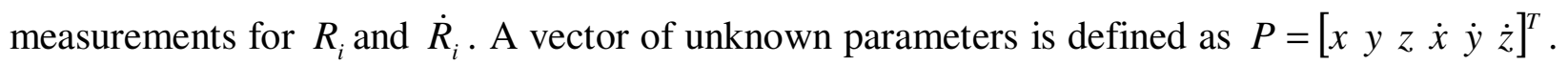
Also, an error vector is defined as:

$$
E_{k}=\left\{\begin{array}{c}
\left(x-x_{s_{1}}\right)^{2}+\left(y-y_{s_{1}}\right)^{2}+\left(z-z_{s_{1}}\right)^{2}-R_{1}^{2} \\
\vdots \\
\left(x-x_{s_{n}}\right)^{2}+\left(y-y_{s_{n}}\right)^{2}+\left(z-z_{s_{n}}\right)^{2}-R_{n}^{2}
\end{array}\right\}
$$

which must be minimized. Solutions to these nonlinear algebraic equations are generated by a standard Newton-Raphson method with a backtracking line search algorithm:

$$
P_{k+1}=P_{k}-\alpha_{k} J_{k}^{-1} E_{k}
$$

The line search optimizer termination criteria are:

1. 2 -norm $\left(\alpha_{k} J_{k}^{-1} E_{k}\right) \leq 0.0001$

2. $2-\operatorname{norm}\left(E_{k}\right) \leq\left(0.9 * 2-\operatorname{norm}\left(E_{1}\right)\right)$

3. $\alpha_{\mathrm{k}} \leq(1 / 2)^{\wedge}(44)$

To simulate an actual gun launch, the navigation algorithm is started after one second of flight time. This demonstrates the typical amount of time for the receiver to settle and produce actual navigation solutions. Note that an initial guess must be made as to the current position and velocities, which is done by fitting a second-order polynomial to the previous ten calculated navigation solutions. Also, trilateration is employed as a backup should the navigation solution or initial guess not meet certain criteria ${ }^{[12]}$.

Once the position solution is obtained from the range equations, the velocity solution can be obtained via linear least squares: 


$$
\left[\begin{array}{ccc}
\frac{x-x_{s_{1}}}{R_{1}} & \frac{y-y_{s_{1}}}{R_{1}} & \frac{z-z_{s_{1}}}{R_{1}} \\
\frac{x-x_{s_{n}}}{R_{n}} & \frac{y-y_{s_{n}}}{R_{n}} & \frac{z-z_{s_{n}}}{R_{n}}
\end{array}\right]\left\{\begin{array}{c}
\dot{x} \\
\dot{y} \\
\dot{z}
\end{array}\right\}=\left\{\begin{array}{c}
\dot{R}_{1} \\
\vdots \\
\dot{R_{n}}
\end{array}\right\}
$$

In order to simulate decreased signal integrity via obstruction due to buildings, trees, and other structures, a line of sight algorithm is utilized to determine whether or not the projectile has a direct line of sight to the signal source or not. If there is not a direct line of sight, the signal is not used in determining the navigation solution. This simulates logic on the smart projectile's receiver that if the signal strength dropped below some threshold, then the signal is not used to calculate the current navigation solution. An example of the line of sight algorithm is shown in Figure 2.

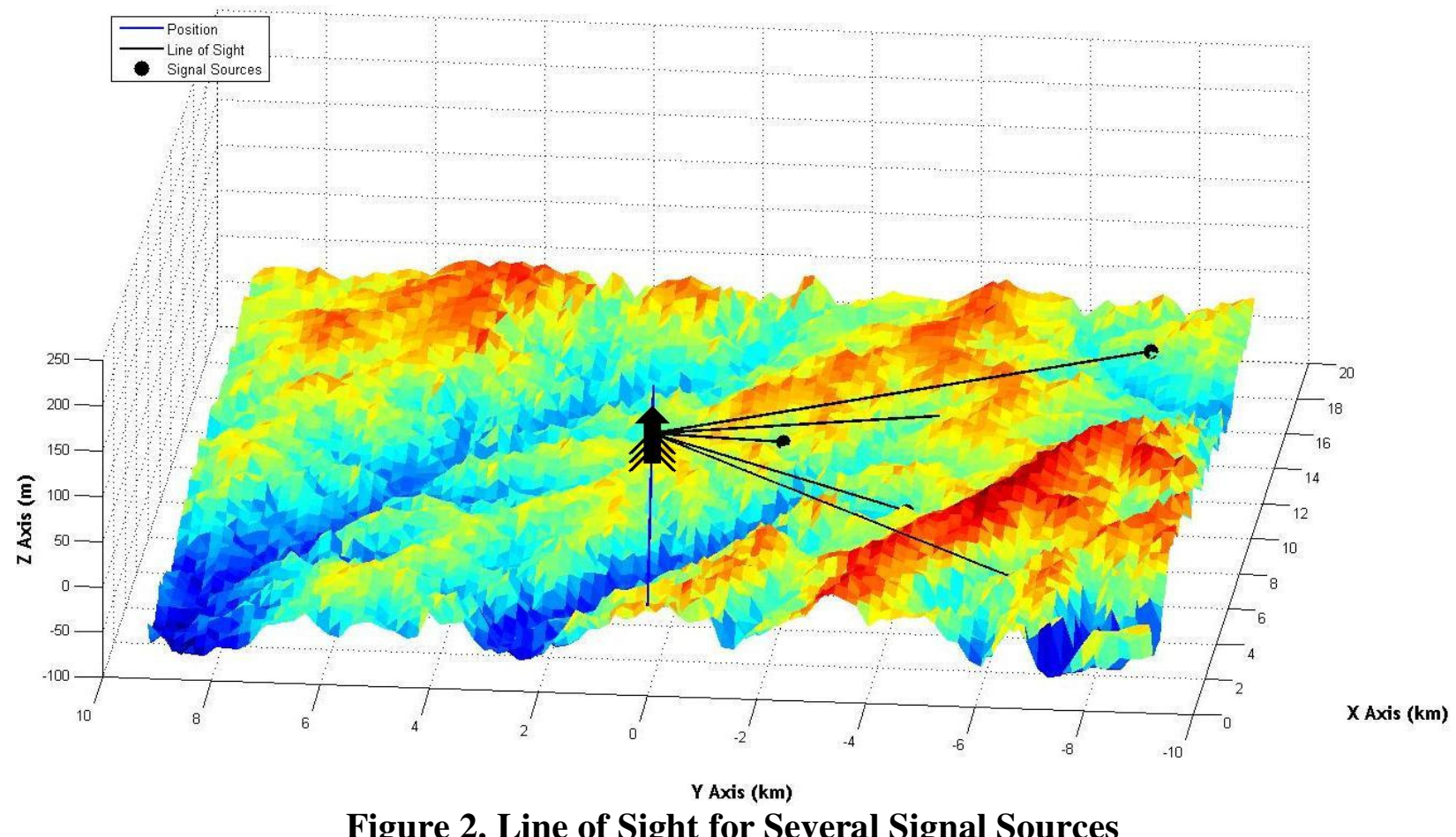




\section{CHAPTER 4 - EXAMPLE RESULTS}

The M549 projectile used to examine the effectiveness of the navigation solution is a representative indirect fire, spin stabilized projectile with a weight of $94.9 \mathrm{lbs}$ and length of 155 mm. Typical muzzle velocities are around $826 \mathrm{~m} / \mathrm{s}$ with a spin rate of about $1,674 \mathrm{rad} / \mathrm{s}$. At a quadrant elevation of $0.2239 \mathrm{rad}$, the M549 projectile will travel roughly $14 \mathrm{~km}$ with a maximum altitude of 1,120 m and an approximate cross range deviation of $88 \mathrm{~m}$ with 30 seconds of flight time. In order to generate these values and a nominal trajectory, a validated six degree of freedom model, by the name of BOOM, was used.

Several initial studies were performed in order to first understand and validate the navigation solution. The first case employed the use of 200 ground based signals centered around the impact point of the nominal trajectory. In order to generate the signal locations, elevation data was acquired from the National Elevation Dataset (NED) to represent actual mission environments. For this first study, elevation dataset for the Bonneville Salt Flats was used because it only varied by $6 \mathrm{~m}$ over the entire $18.3 \mathrm{~km}$ by $18.3 \mathrm{~km}$ section of land. Figure 3 shows the location of the 200 signals plotted on the 3D elevation data for the Bonneville Salt Flats. The error metrics used were $\sigma=0.0001$ and $b=0.3 \mathrm{~m}$, which are representative of AM and or lower frequency eLORAN signals. These values along with additional signal error levels can be seen in Table 1. 


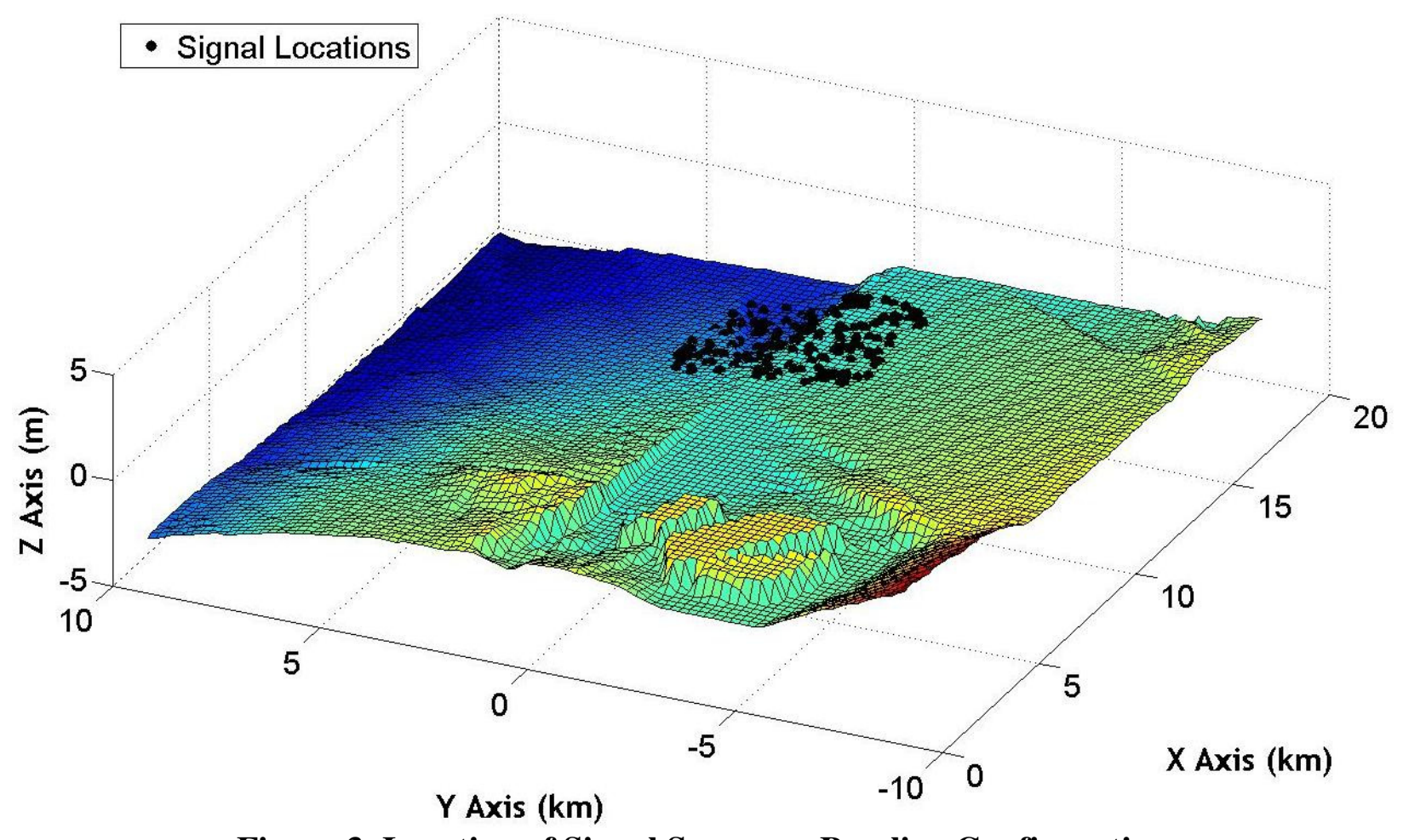

Figure 3. Location of Signal Sources - Baseline Configuration

Figures $4-6$ show the navigation solution for position using the proposed algorithm. Notice that the estimated position is only plotted every second, but in fact is calculated every 0.01 seconds. From these three plots, one can deduce that there is a direct correlation between accuracy of navigation solution and distance from the concentration of signal sources. Figure 7 provides the $3 \mathrm{D}$ trajectory of the projectile along with the location of the 200 ground based RF signals. To better demonstrate the correlation between distance from signal sources and accuracy, the residual of the position calculations are plotted in Figure 8, where the residual is the absolute value of the difference between the actual position and estimated position. Note that the $\mathrm{X}$ and $\mathrm{Y}$ residuals follow a similar trend and decrease as the projectile approaches the target and concentration of signal sources. However, the $\mathrm{Z}$ residual tends to deviate from this trend near the target, and will be further discussed below. Figure 9 graphs the velocity residuals throughout 
the entire trajectory, and as expected, the velocity residuals follow similar trends as the position residuals.

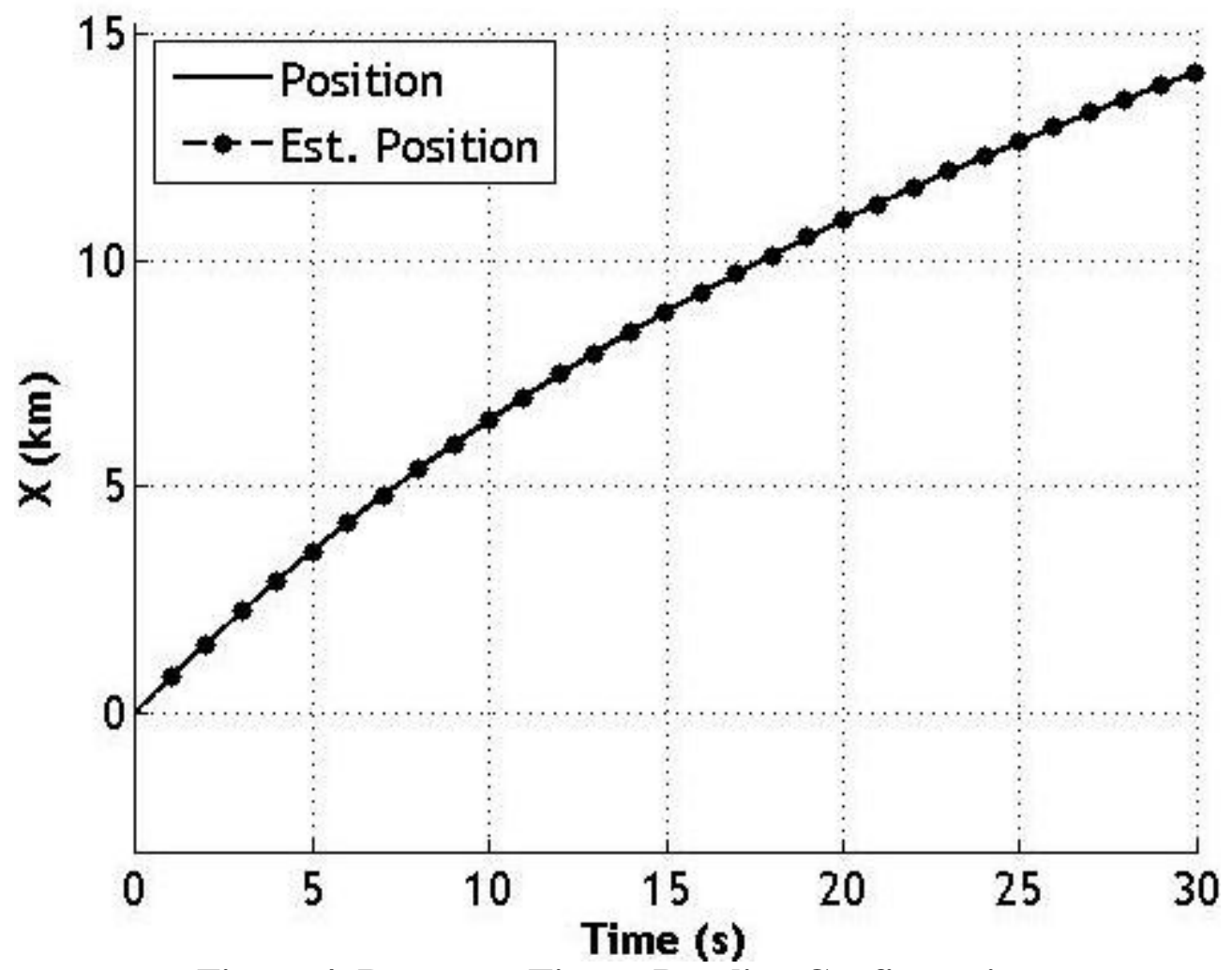

Figure 4. Range vs. Time - Baseline Configuration 


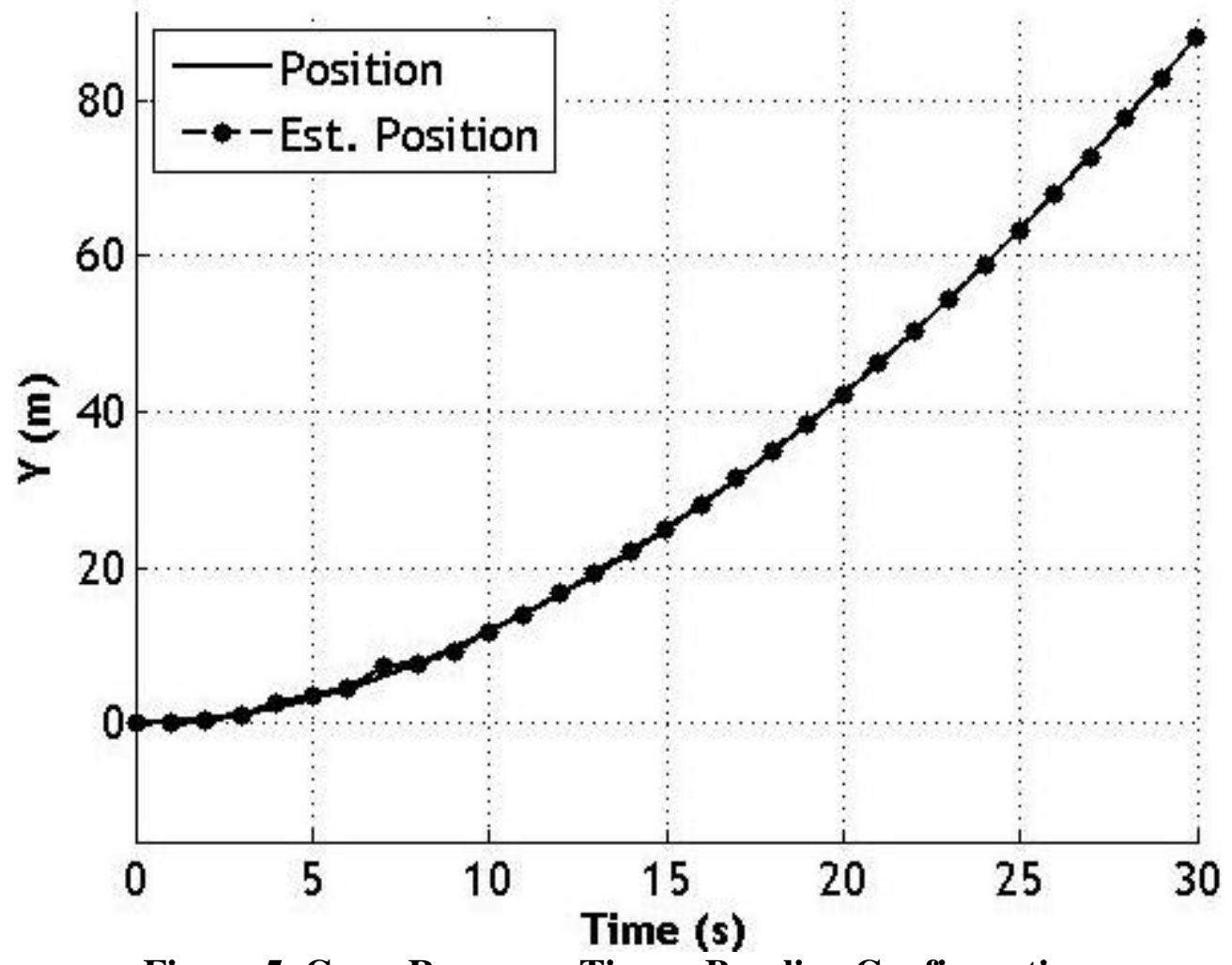

Figure 5. Cross Range vs. Time - Baseline Configuration 


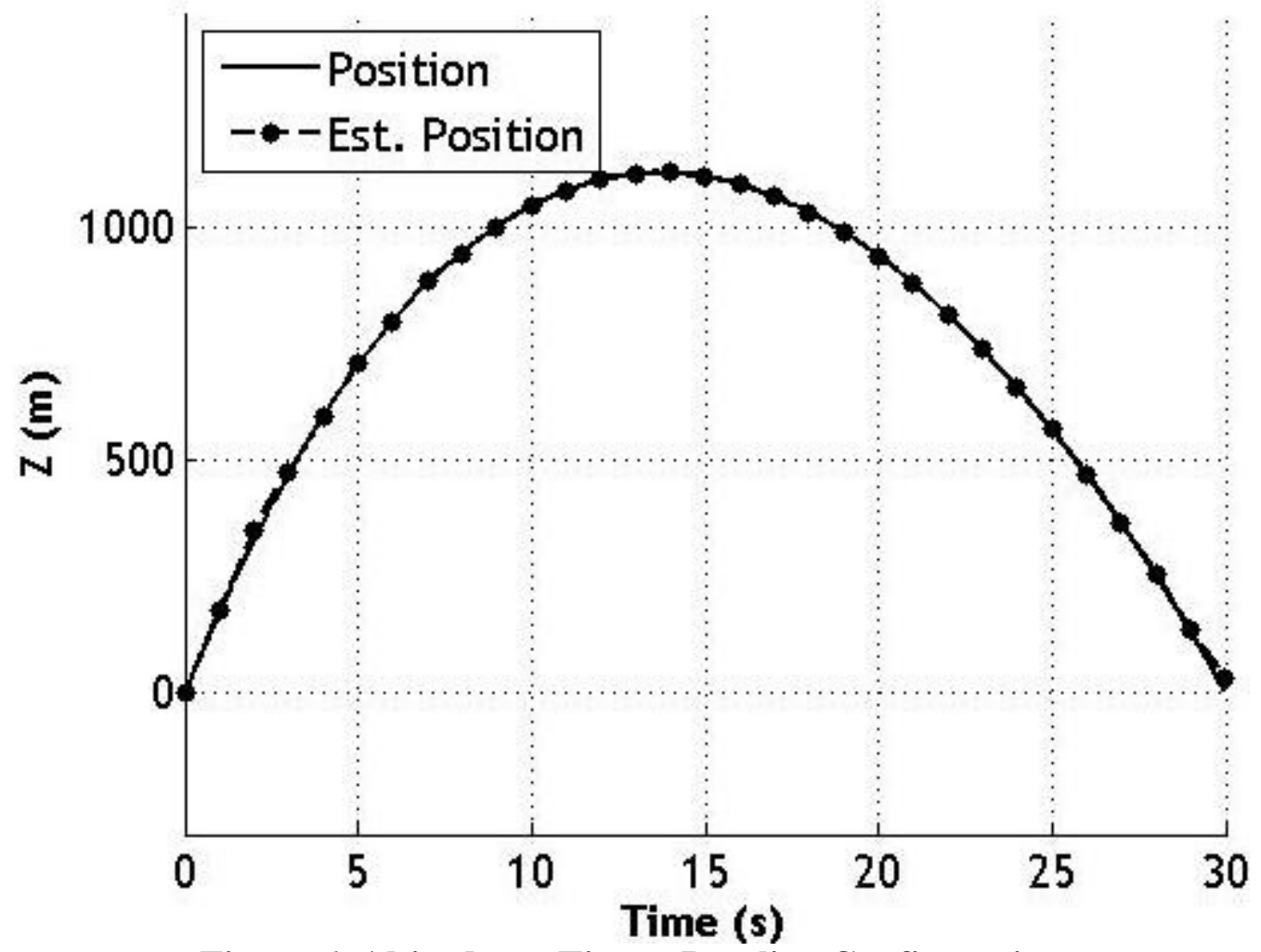

Figure 6. Altitude vs. Time - Baseline Configuration 


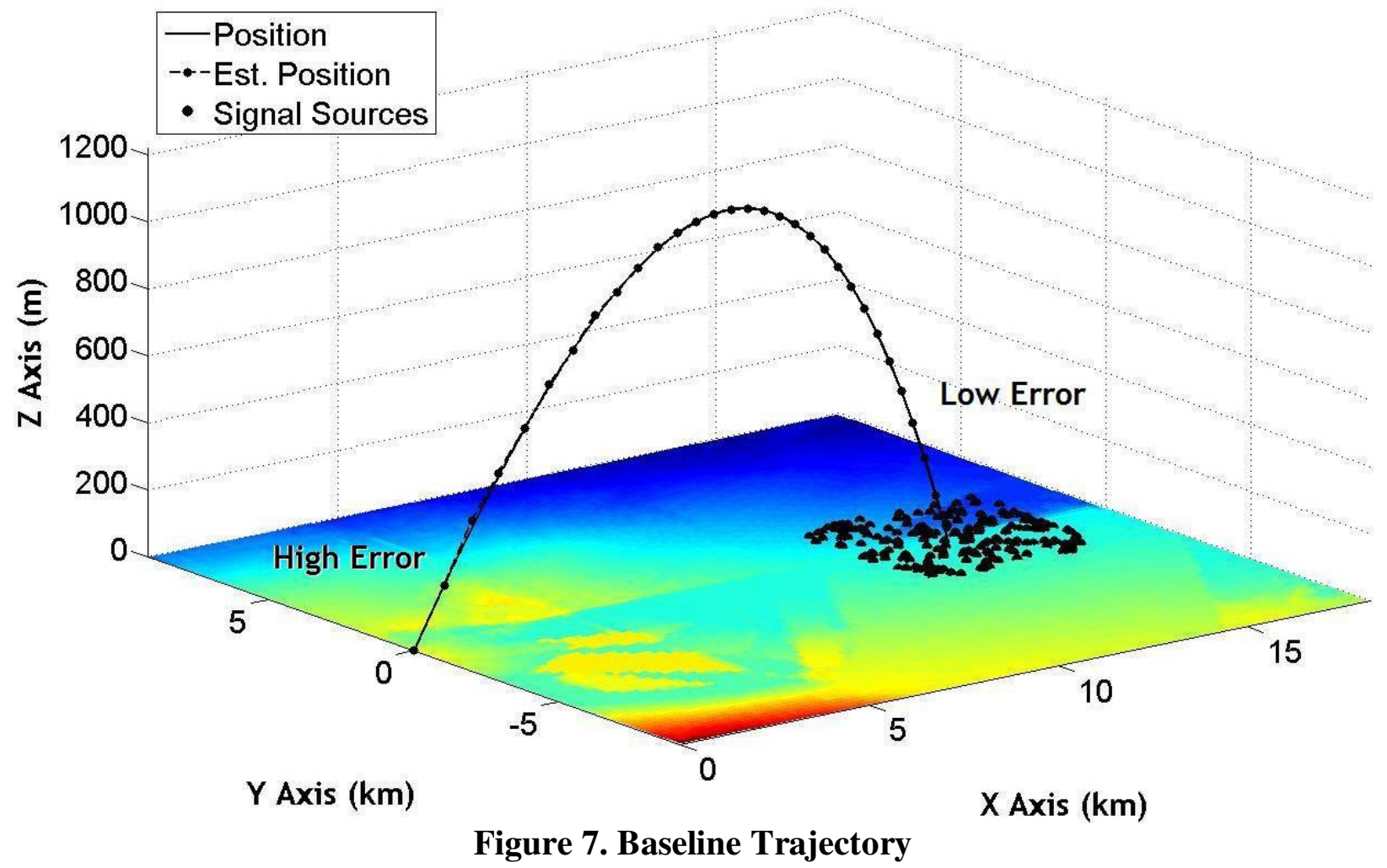



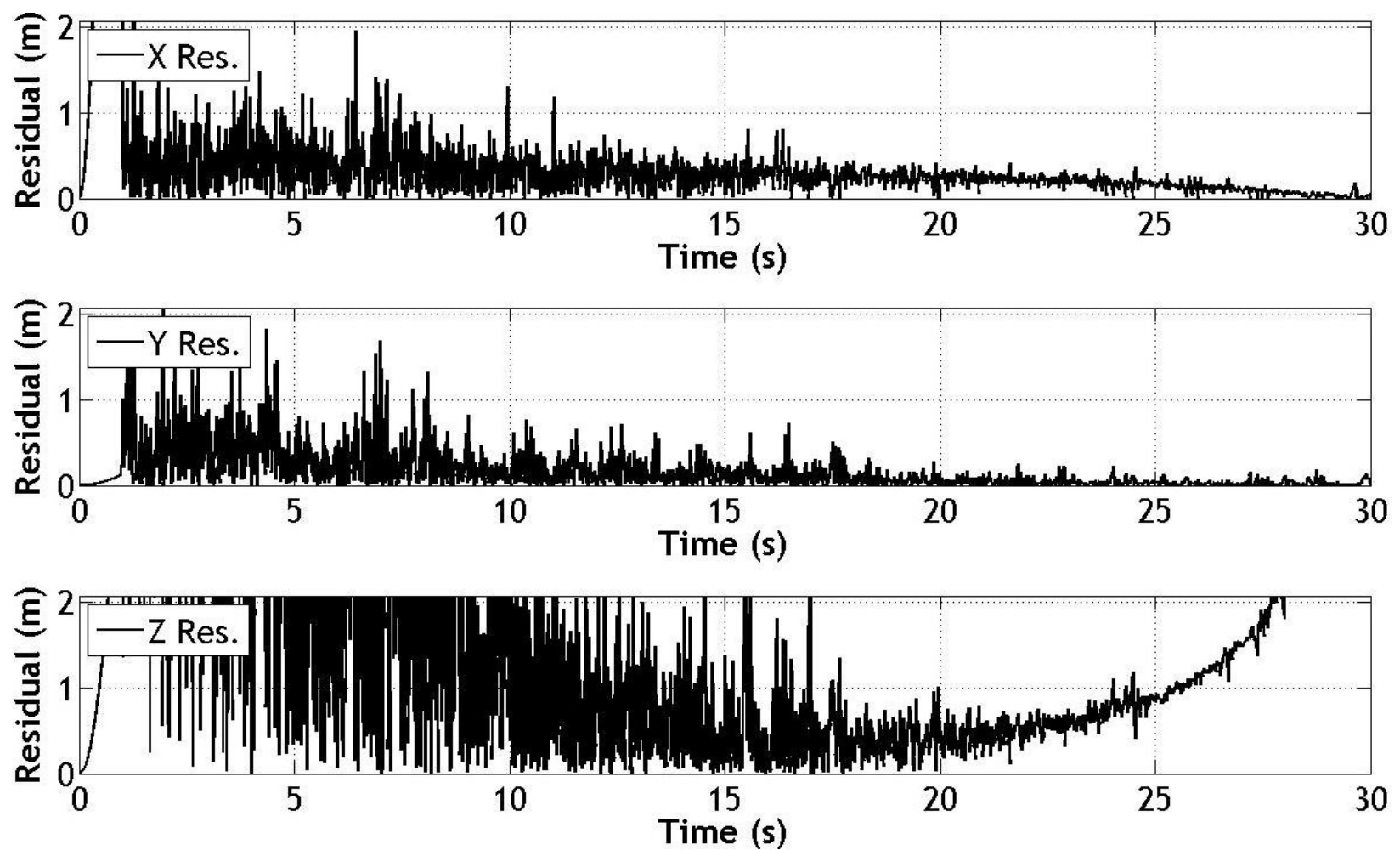

Figure 8. Position Residuals vs. Time - Baseline Configuration 

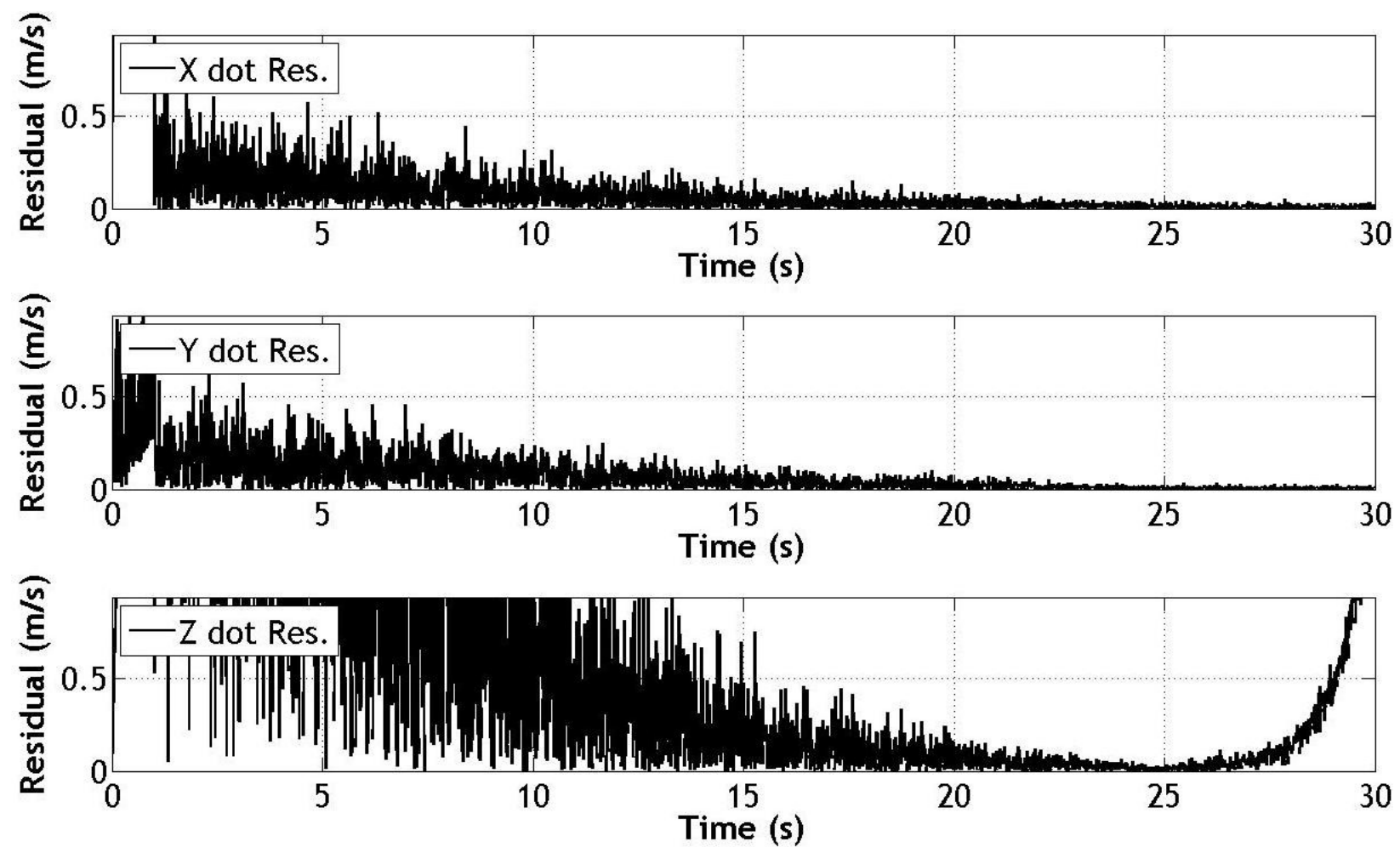

Figure 9. Velocity Residuals vs. Time - Baseline Configuration

The Monte Carlo consisted of 1,000 different cases, each with different initial conditions that were generated to explore characteristics of the navigation solution. In order to suppress the output of the navigation solution, a minimal set of navigation solutions are presented during each iteration, which corresponded to an output at $0 \%, 10 \%, 20 \% \ldots 100 \%$ of the trajectory. This simulation consisted of $50 \mathrm{RF}$ ground based signal sources, and the following error levels, $\sigma=$ 0.0001 and $b=0.3 \mathrm{~m}$. Figure 9 represents the 3D navigation solution along with the signal source locations. The blocks at $3 \mathrm{~s}, 15 \mathrm{~s}$ and $30 \mathrm{~s}$ on Figure 10 are there to demonstrate three main areas of focus. These three segments of the trajectories will be used to further develop conclusions based on the parametric trade studies. 


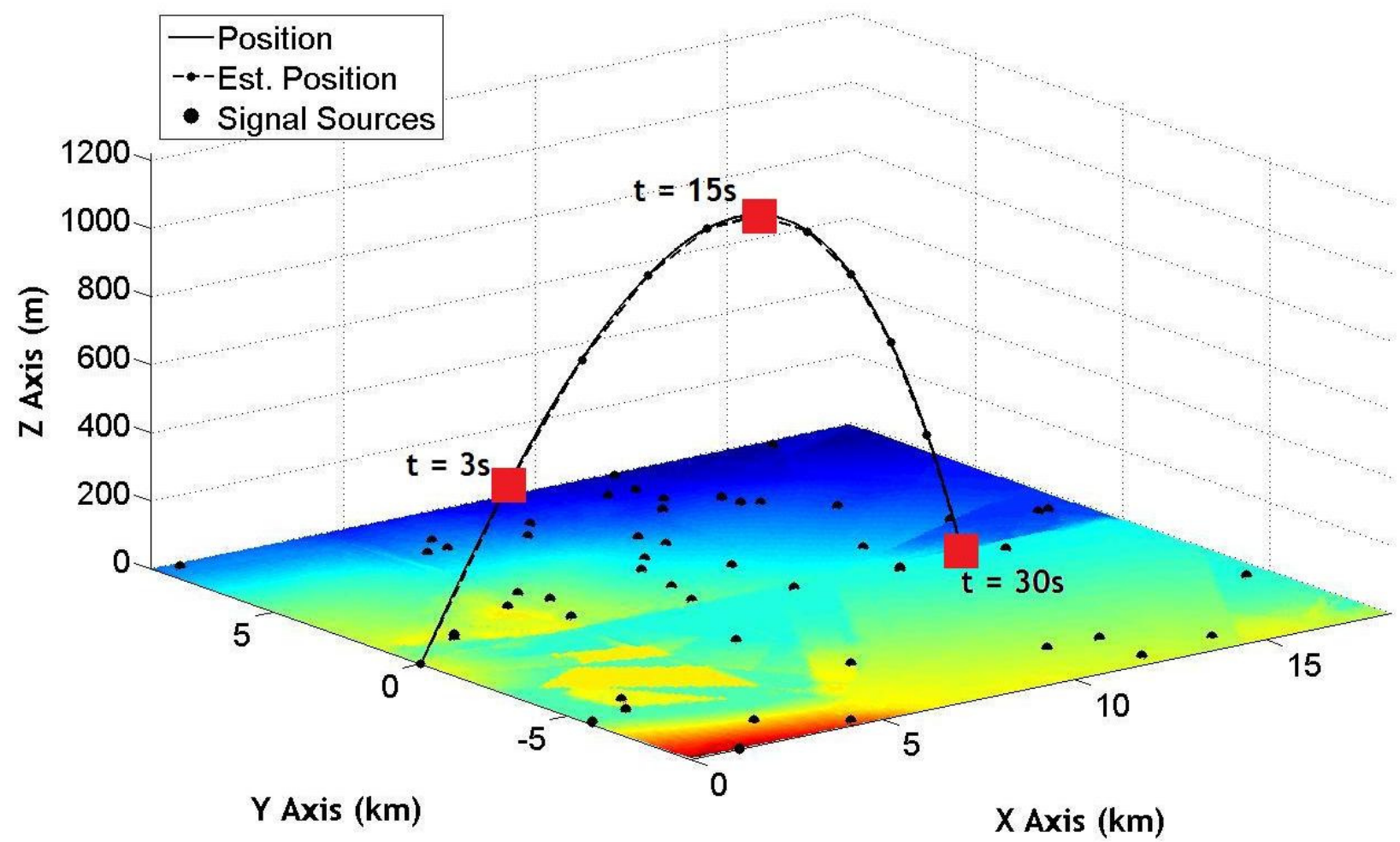

Figure 10. 3D Trajectory with Elevation Data and Areas of Focus: 3s, 15s and 30s

Figure $11-13$ present a normalized histogram of the position calculations at $3 \mathrm{~s}, 15 \mathrm{~s}$ and $30 \mathrm{~s}$, along with the actual position and mean of the estimated position for each iteration of the Monte Carlo simulation. As seen in the previous nominal study, the $\mathrm{X}$ and $\mathrm{Y}$ position behave very similarly, with the estimated altitude becoming worse near the target. 

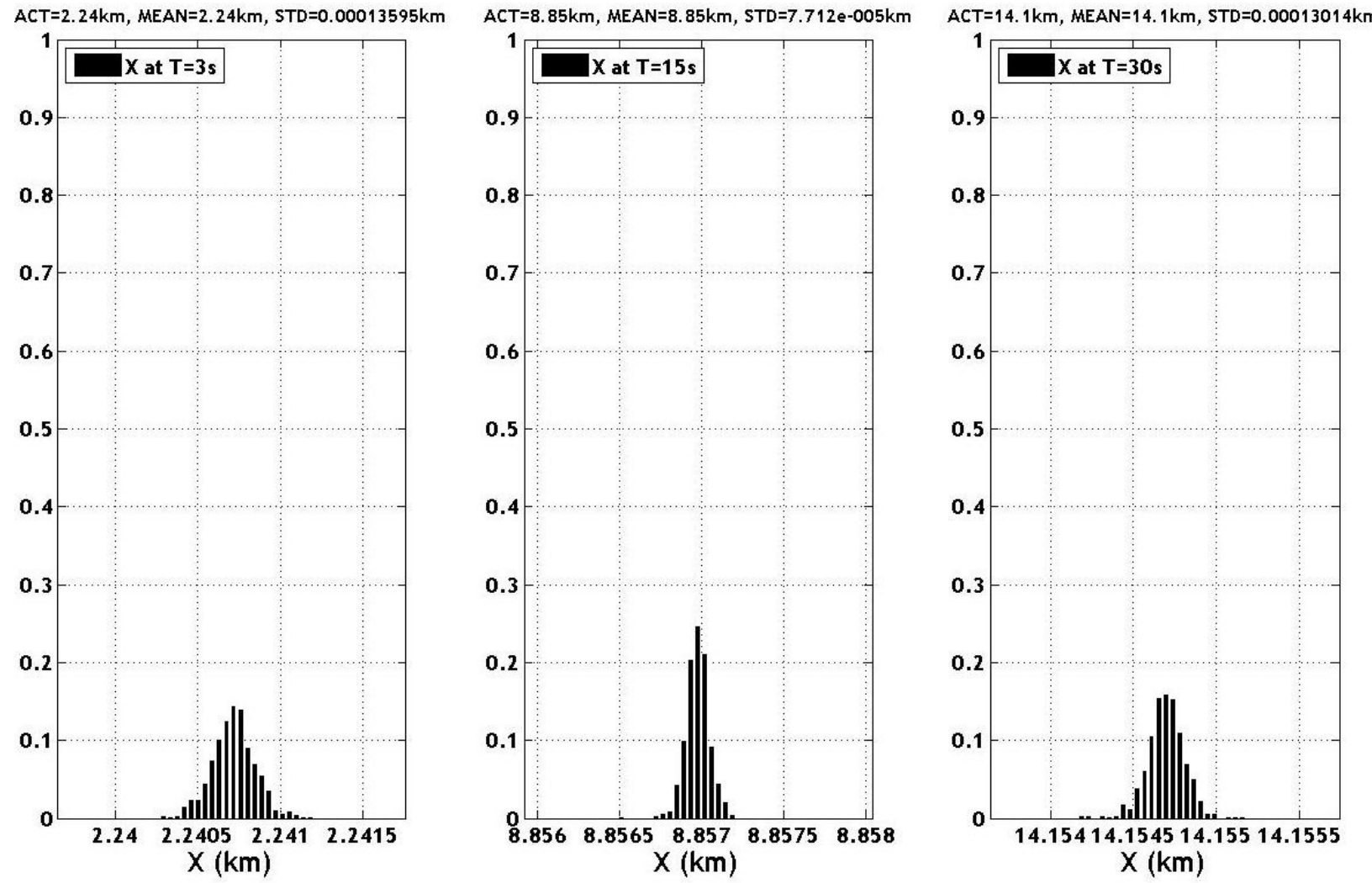

Figure 11. Histograms of $X$ Calculated at $t=3 \mathrm{~s}, 15 \mathrm{~s}$ and 30s - Monte Carlo Simulation 

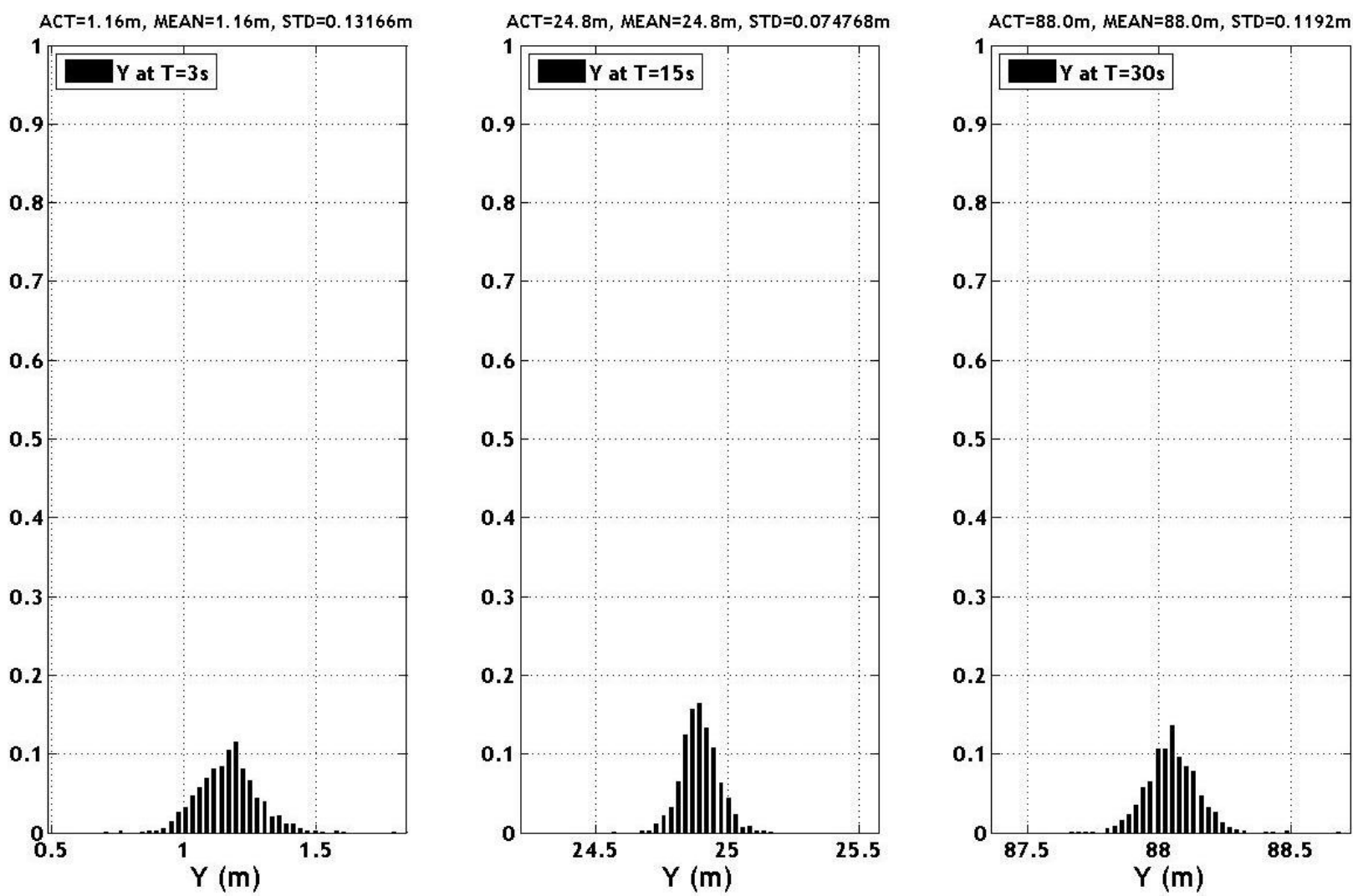

Figure 12. Histograms of $Y$ Calculated at $t=3 s, 15 s$ and 30s - Monte Carlo Simulation 

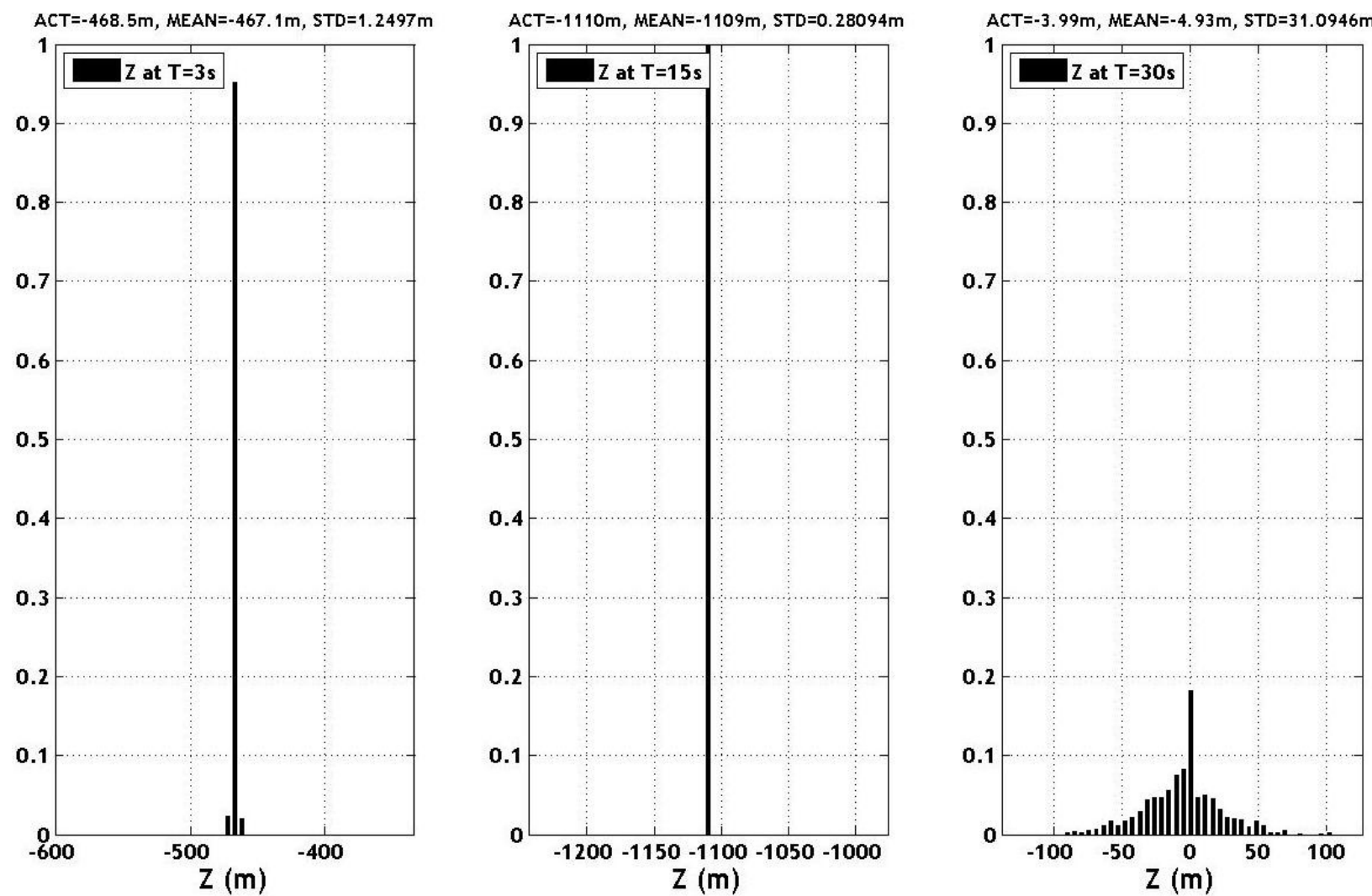

Figure 13. Histograms of $\mathrm{Z}$ Calculated at $\mathrm{t}=3 \mathrm{~s}, 15 \mathrm{~s}$ and $30 \mathrm{~s}-$ Monte Carlo Simulation 


\section{CHAPTER 5 - PARAMETRIC TRADE STUDIES}

Based on the initial case studies, various characteristics were identified and will be studied separately. In order to effectively compare the studies against one another, a nominal set of metrics were determined. The first two deal with error levels on the range and range rate, $\sigma=$ 0.0001 and $b=0.3 \mathrm{~m}$. The number of ground based RF signals was set to nominally be 50 , with an error in positioning of $1 \mathrm{~m}$. Typically, unless otherwise stated, the signal sources were allowed to be placed over the entire $18.3 \mathrm{~km}$ by $18.3 \mathrm{~km}$ terrain map. Additionally, the trade studies were evaluated on three different environments, using actual elevation data. These were used to simulate flat land, mountains and an urban landscape. 3D Surface plots for these elevation data sets can be seen in Figures $14-16$. The Bonneville Salt Flats in Figure 14 had a maximum altitude of $3.7 \mathrm{~m}$ and minimum altitude of $-2.7 \mathrm{~m}$, which provides very little variation in the altitude of the ground based signals. The opposite is the case for the mountainous terrain of Colorado shown in Figure 15, which had a maximum altitude of $257.2 \mathrm{~m}$ and minimum altitude

of $-356.4 \mathrm{~m}$. The last landscape depicted in Figure 16 is the elevation dataset for the city of Atlanta, which had a maximum altitude of $78 \mathrm{~m}$ and a minimum altitude of $-38.4 \mathrm{~m}$. 
Flat Land: Max Height $=3.7 \mathrm{~m} \&$ Min Height $=-2.7 \mathrm{~m}$

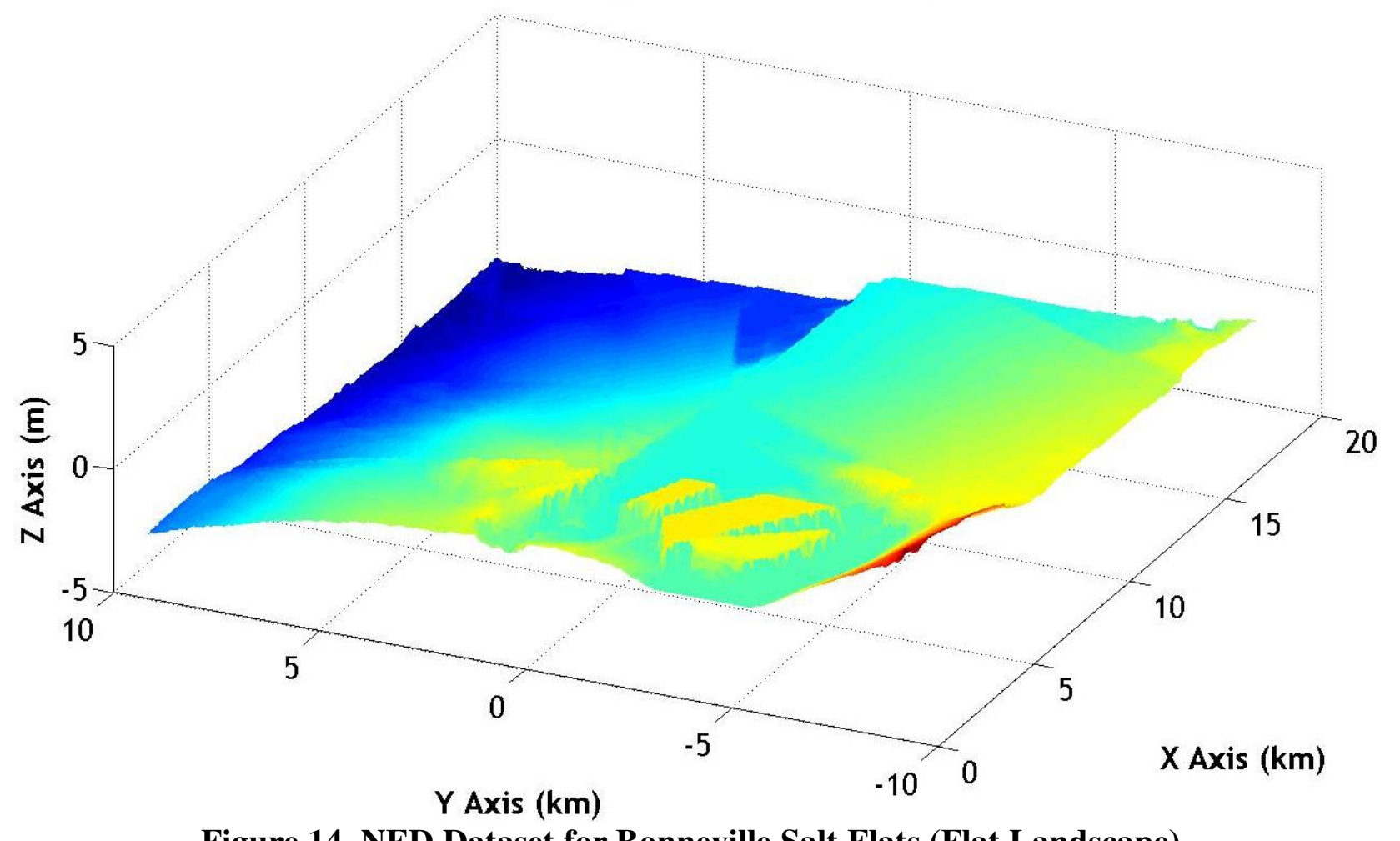

Figure 14. NED Dataset for Bonneville Salt Flats (Flat Landscape) 


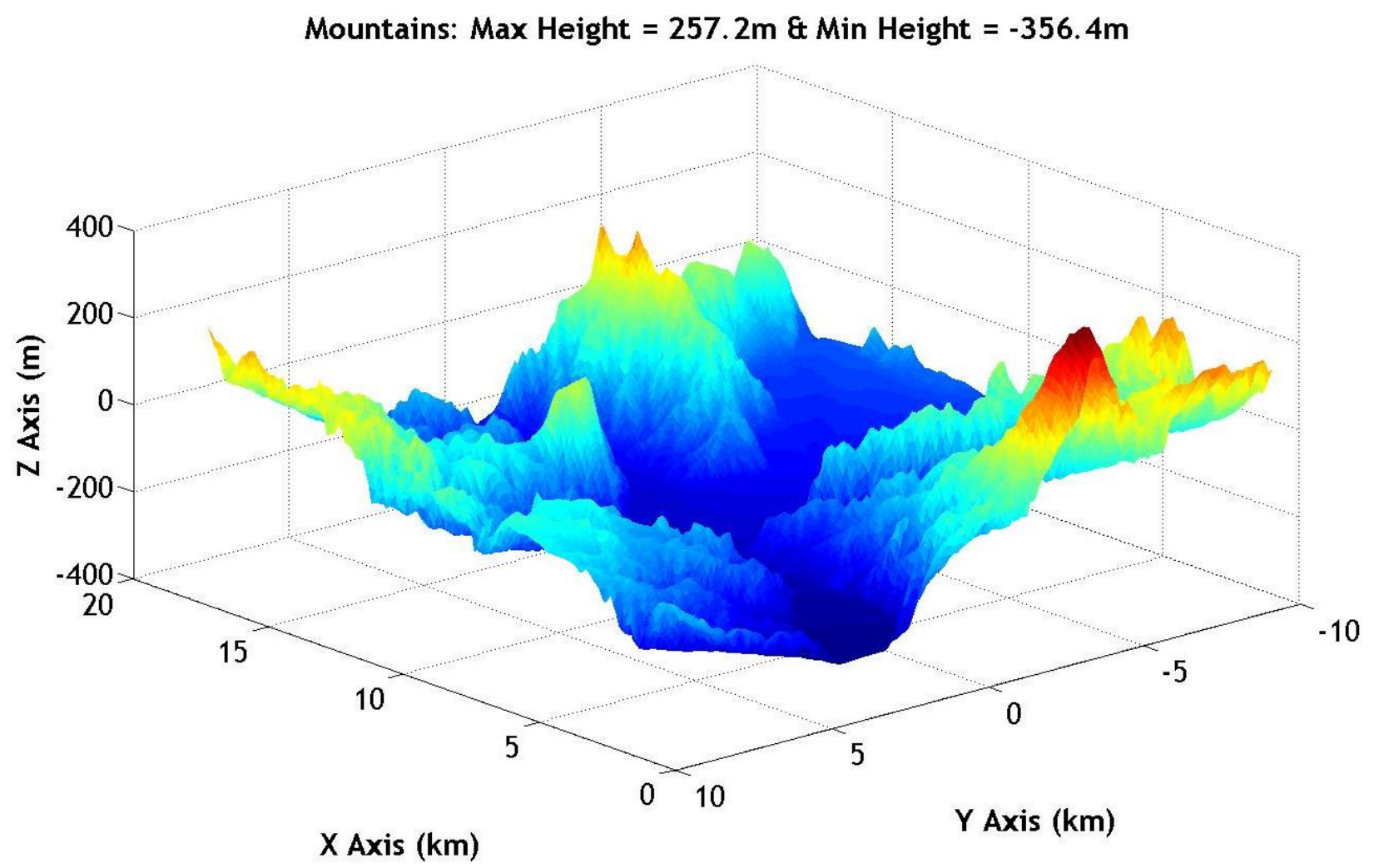

Figure 15. NED Dataset for Colorado Mountains (Mountainous Landscape) 


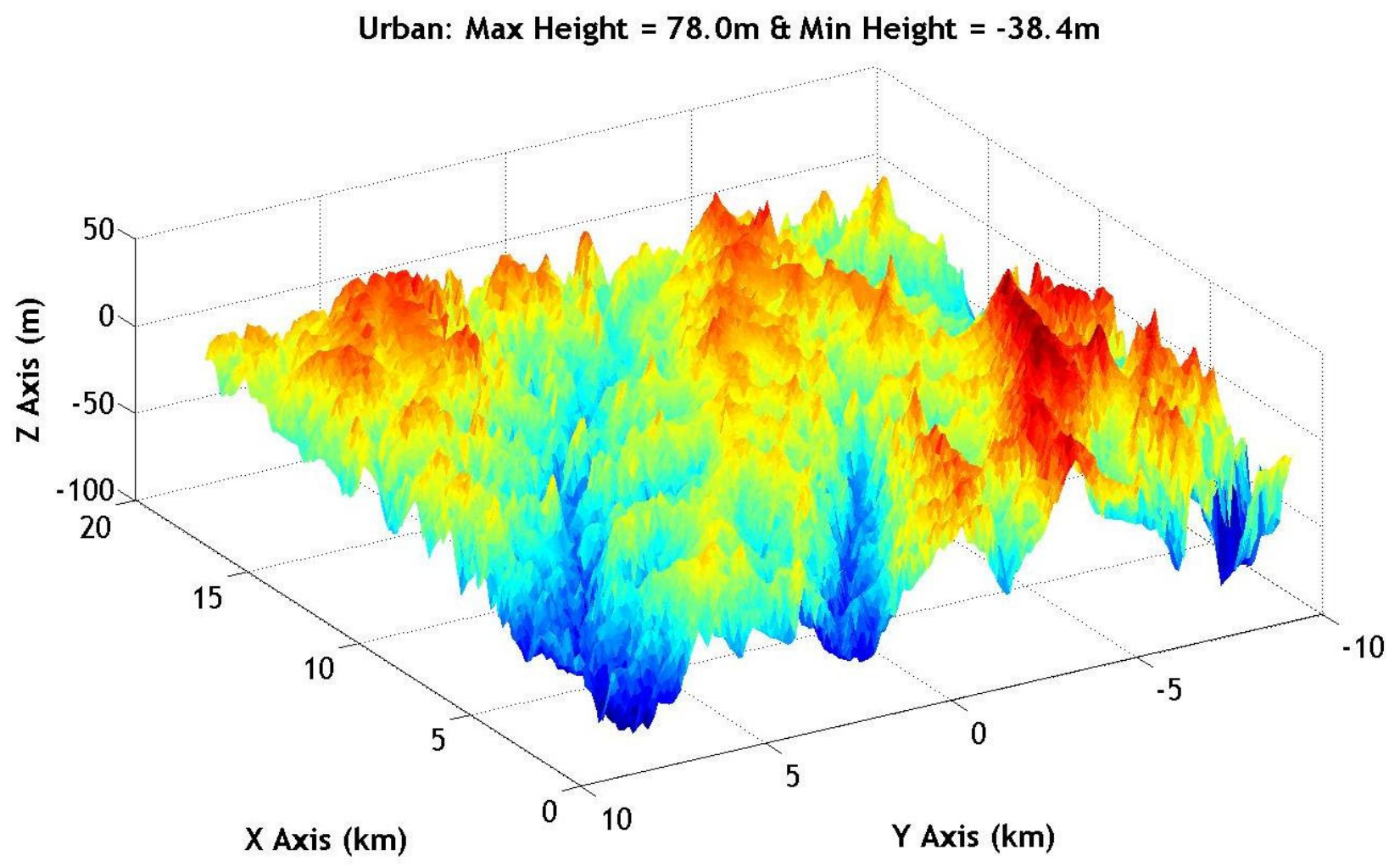

Figure 16. NED Dataset for Atlanta (Urban Landscape)

The accuracy of the navigation solutions are analyzed using a 95\% confidence sphere. In order to develop these confidence spheres, the residuals for the estimated position were combined in to a radius and the metric reported was the radius which encompassed $95 \%$ of all the navigation solutions at that point in the trajectory. The same metric was used to describe the accuracy of the estimated velocity. Figure 17 is a graphical depiction of the confidence sphere. 


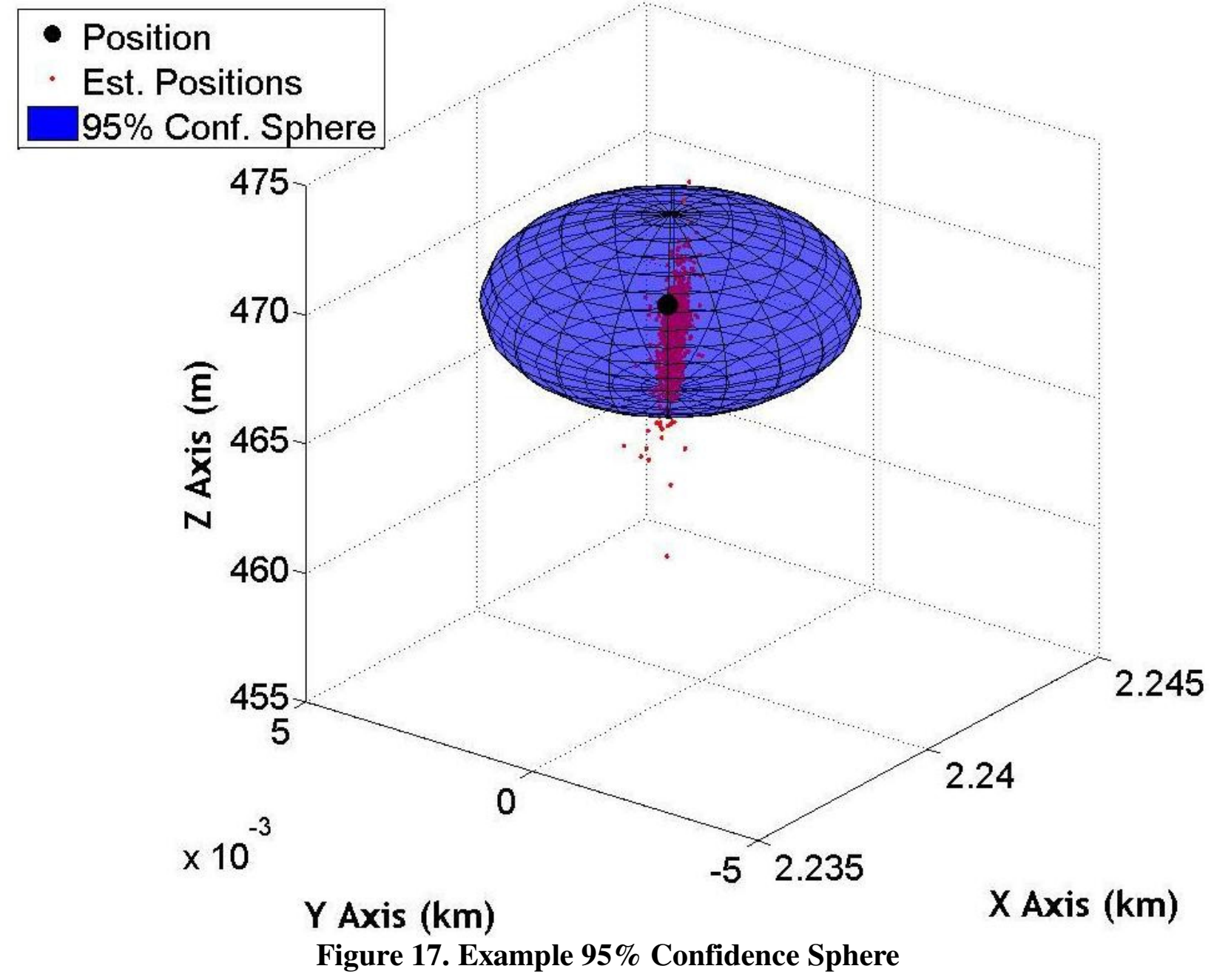


The effect of the RF ground based signals grid size is explored in Figures 18 and 19. Six different square grid sizes were evaluated, at sizes of $0.3 \mathrm{~km}, 1.5 \mathrm{~km}, 3.0 \mathrm{~km}, 6.1 \mathrm{~km}, 9.1 \mathrm{~km}$ and $12.2 \mathrm{~km}$. Each grid was located at the center of the trajectory. Figure 18 displays the confidence spheres for estimating the position at 3s, 15s and 30s. As expected, by increasing the grid size, the accuracy of the navigation solution improves with the best performance arising from the mountainous landscape. At time equal to $15 \mathrm{~s}$, the projectile is directly over the center of the grids, which is why little improvement is noticed by increasing grid size. When the projectile is about to impact the ground, there is still an improvement for the mountain and urban landscapes, but not for the flat ground. This phenomenon is attributed to the fact that there is little variation in altitude of the signal sources. Identical trends are seen in Figure 19 for estimating velocities, with largest confidence spheres occurring near the target. 

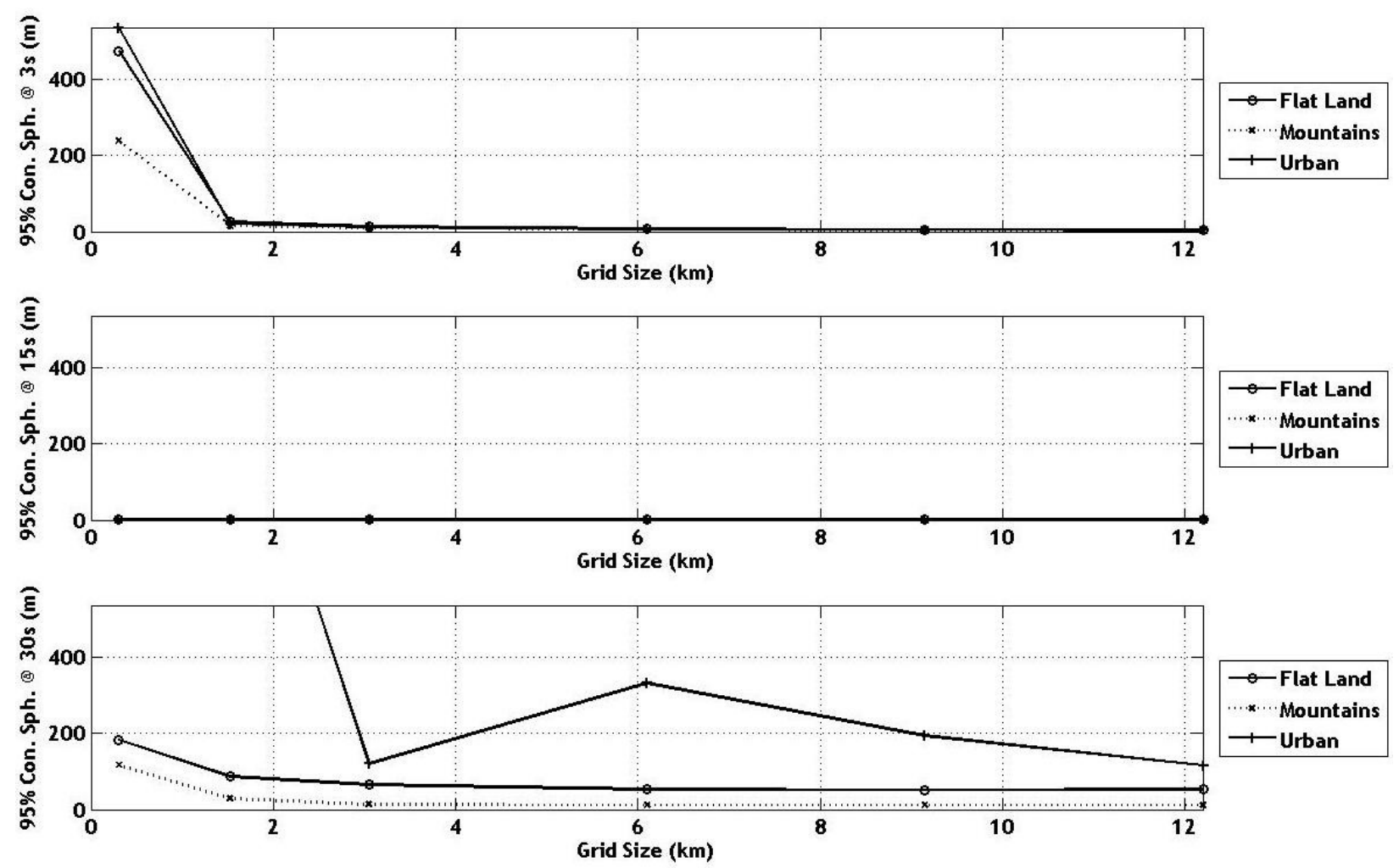

Figure 18. 95\% Confidence Position Spheres vs. Grid Size for 3s, 15s and 30s 

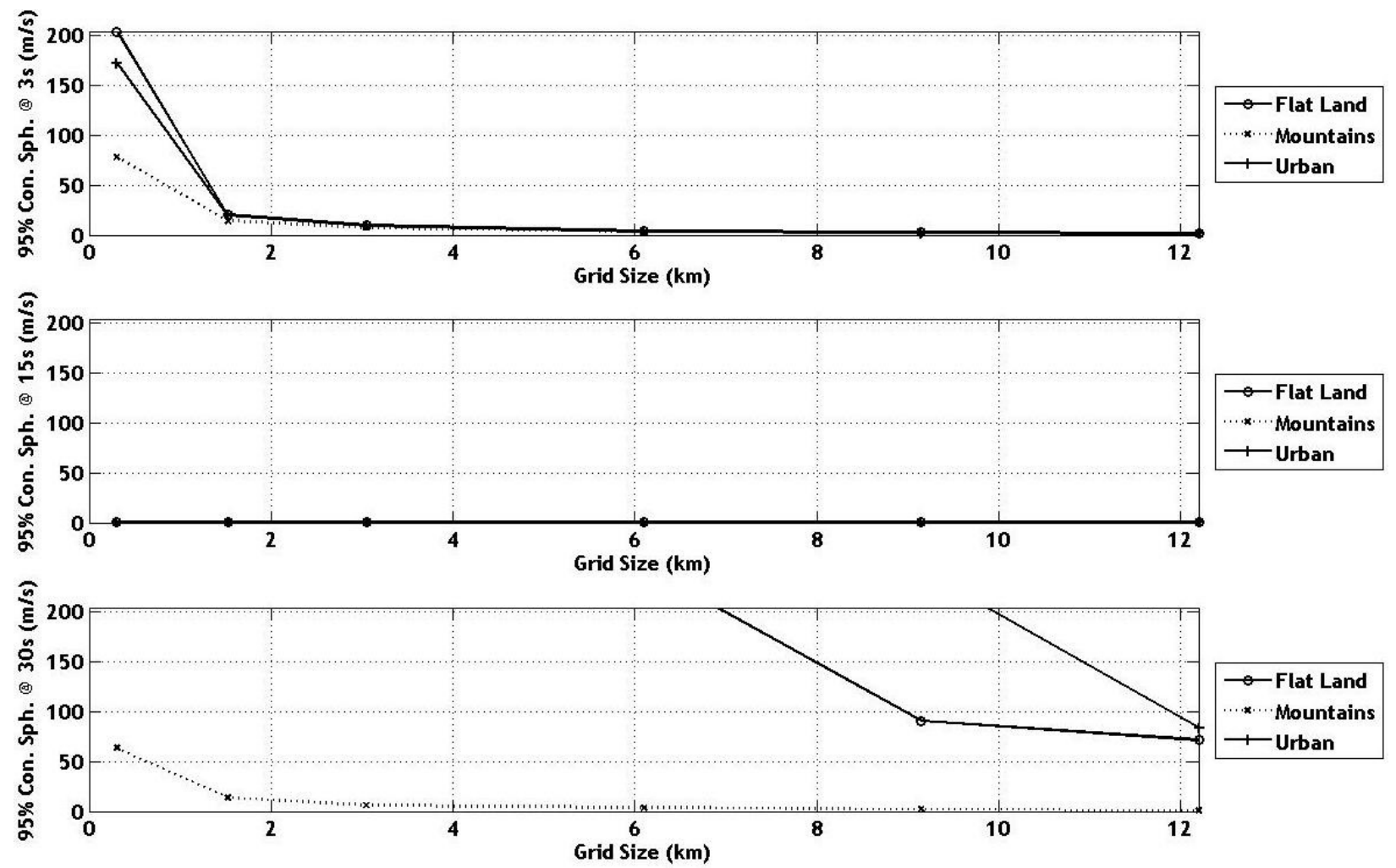

Figure 19. 95\% Confidence Velocity Spheres vs. Grid Size for 3s, 15s and 30s

In order to study the density of ground based signals of opportunity, a trade study was performed varying the number of signals available. The numbers of signals utilized were 25,50 , 100, 150, 200, 300, 400 and 500. Figure 20 displays the confidence spheres for estimated position and interestingly at $\mathrm{t}=3 \mathrm{~s}$ the accuracy is directly correlated to the number of signals of opportunity available but only to a certain level. Beyond 200 signal sources accuracy asymptotically approaches a limit. This same trend can be seen at $t=15 \mathrm{~s}$, but on a smaller scale. However, accuracy near the target is degraded by the loss of line of sight, reduced visibility and decreased signal sources. Similar trends can be seen in Figure 21, which plots the velocity confidence spheres as a function of the number of initial signal sources. 

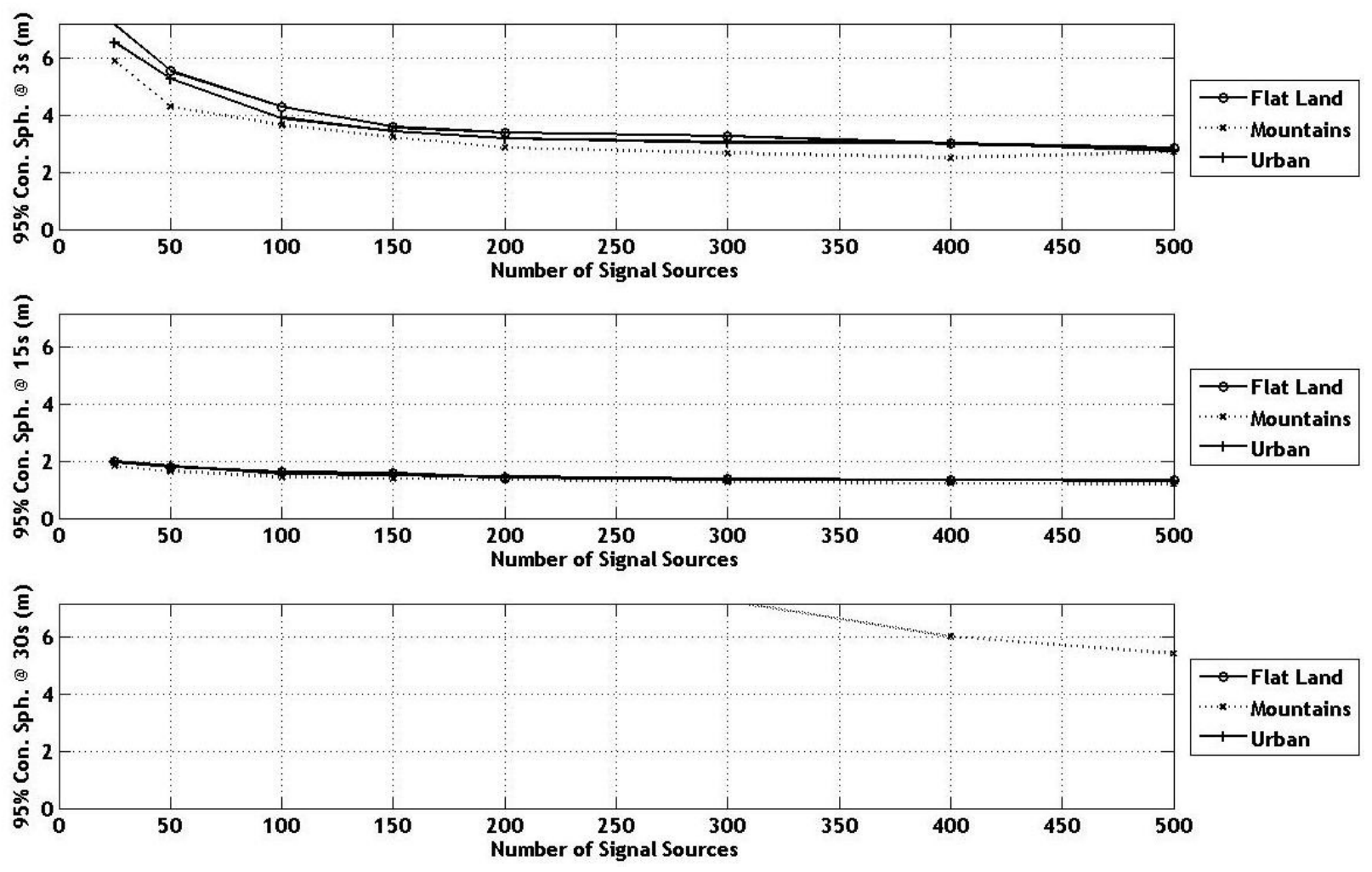

Figure 20. $95 \%$ Confidence Position Spheres vs. Number of Signal Sources for 3s, 15s and 30s 

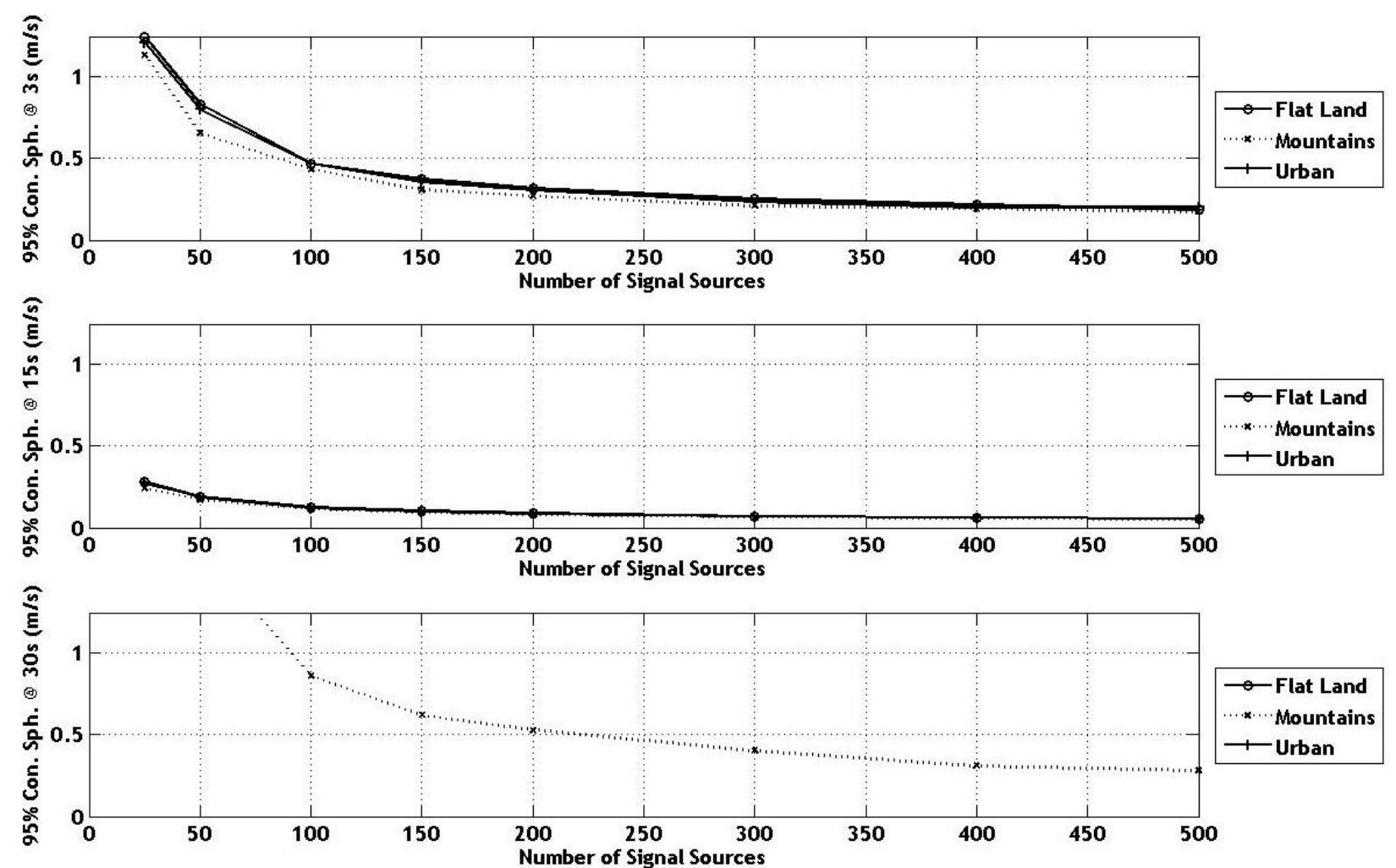

Figure 21. 95\% Confidence Velocity Spheres vs. Number of Signal Sources for $3 \mathrm{~s}$, 15s and 30s

Ground location of the signals relative to the trajectory plays an important role in accuracy, which is why a parametric trade study looked at the effect of the signal sources location on accuracy of the navigation solution. Four main signal locations were selected for this parametric trade study and these were located near the muzzle, directly east of the middle of the trajectory, directly west of the middle of the trajectory and centered around the impact point. 50 signal sources were used here in a $6.1 \mathrm{~km}$ by $6.1 \mathrm{~km}$ grid for all three elevation datasets. The results for this trade study are given in Table 2 . At $t=3 \mathrm{~s}$, the grids located near the gun launch provided the best navigation solution being that these signals are closer to the projectile at this instant in time. When the projectile is 15 seconds into flight, all four locations performed 
relatively the same, which is attributed to the fact that all the signals of opportunity have direct line of sight and that the projectile was relatively the same distance from each signal source grid. Similar to the case when $t=3 \mathrm{~s}$, the navigation solution at $30 \mathrm{~s}$ is best determined by the grid located near the impact point. Based on this trade study, it is apparent that the navigation solution is best calculated when the projectile is directly over the center of the signal source grid.

Table 2. 95\% Confidence Spheres vs. Location of Signal Sources for 3s, $15 \mathrm{~s}$ and $30 \mathrm{~s}$

\begin{tabular}{|c|c|c|c|c|c|c|c|c|c|c|c|c|}
\hline \multirow[b]{2}{*}{ Time } & \multicolumn{4}{|c|}{ FLAT } & \multicolumn{4}{|c|}{ MOUNTAINS } & \multicolumn{4}{|c|}{ URBAN } \\
\hline & South & East & West & North & South & East & West & North & South & East & West & North \\
\hline $3 s$ & 1.0 & 18.1 & 18.7 & 54.4 & 0.8 & 17.0 & 10.4 & 27.6 & 0.9 & 15.4 & 15.1 & 49.9 \\
\hline $15 s$ & 2.2 & 2.5 & 2.4 & 2.8 & 1.7 & 2.2 & 1.9 & 2.4 & 2.2 & 2.3 & 2.3 & 2.5 \\
\hline $30 \mathrm{~s}$ & 36.4 & 13.6 & 12.5 & 1.2 & 16.7 & 7.87 & 7.06 & 0.8 & 30.3 & 12.2 & 11.0 & 1.1 \\
\hline$\widehat{8}$ & 0.2 & 6.9 & 6.8 & 19.4 & 0.2 & 7.1 & 4.0 & 10.9 & 0.3 & 6.7 & 5.8 & 18.6 \\
\hline $15 s$ & 0.9 & 0.4 & 0.4 & 1.2 & 0.8 & 0.3 & 0.3 & 1.0 & 0.9 & 0.4 & 0.3 & 1.1 \\
\hline $30 \mathrm{~s}$ & 6.9 & 1.8 & 1.6 & 0.1 & 3.1 & 1.1 & 0.9 & 0.1 & 6.2 & 1.6 & 1.5 & 0.1 \\
\hline
\end{tabular}

A major factor driving the accuracy of navigation solution is the error level value $\sigma$, which will vary depending on which type signal source is implemented. Experimental values for this error source and the corresponding type of signal is given in Table 1. Using this table as a guide, the effect of error level on accuracy was varied for values of $0.0001,0.0005,0.001,0.005$ and 0.01 , while keeping additional characteristics set to nominal levels. One may expect that as you increase the error levels it may have an exponential effect on the accuracy, and this is not the 
case. At $\mathrm{t}=3 \mathrm{~s}$ and $\mathrm{t}=15 \mathrm{~s}$, there tends to be almost a linear relationship between error levels and confidence spheres, which can be seen in Figure 22. However, near the impact point, the position confidence spheres are corrupted due to the loss of signals and or minimized altitude variation, but the overall trend, that as $\sigma$ increases so does the estimation error, holds true. Figure 23 also demonstrates this property with relation to velocity estimations.
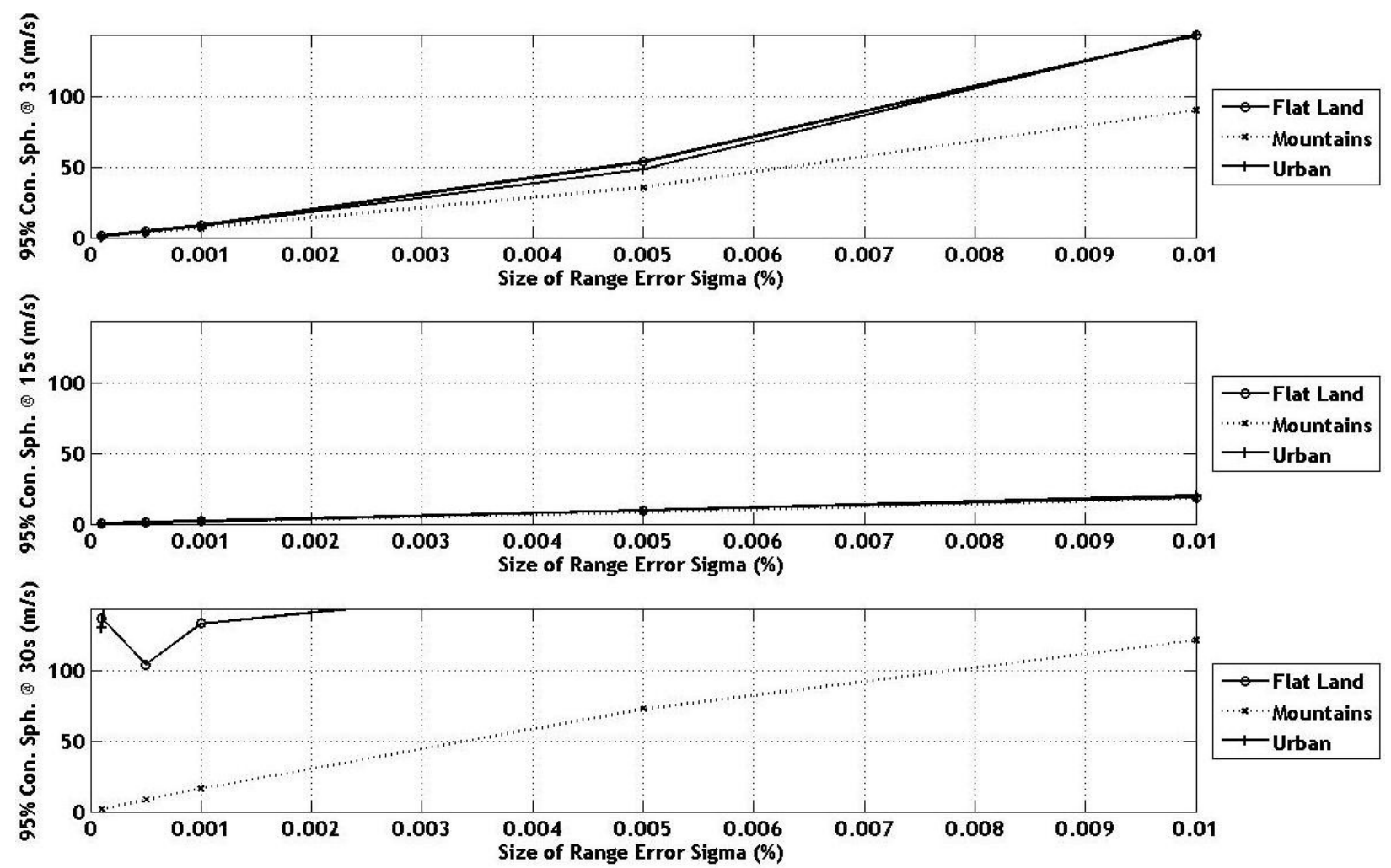

Figure 22. 95\% Confidence Position Spheres vs. Size of Error Level for 3s, 15s and 30s 

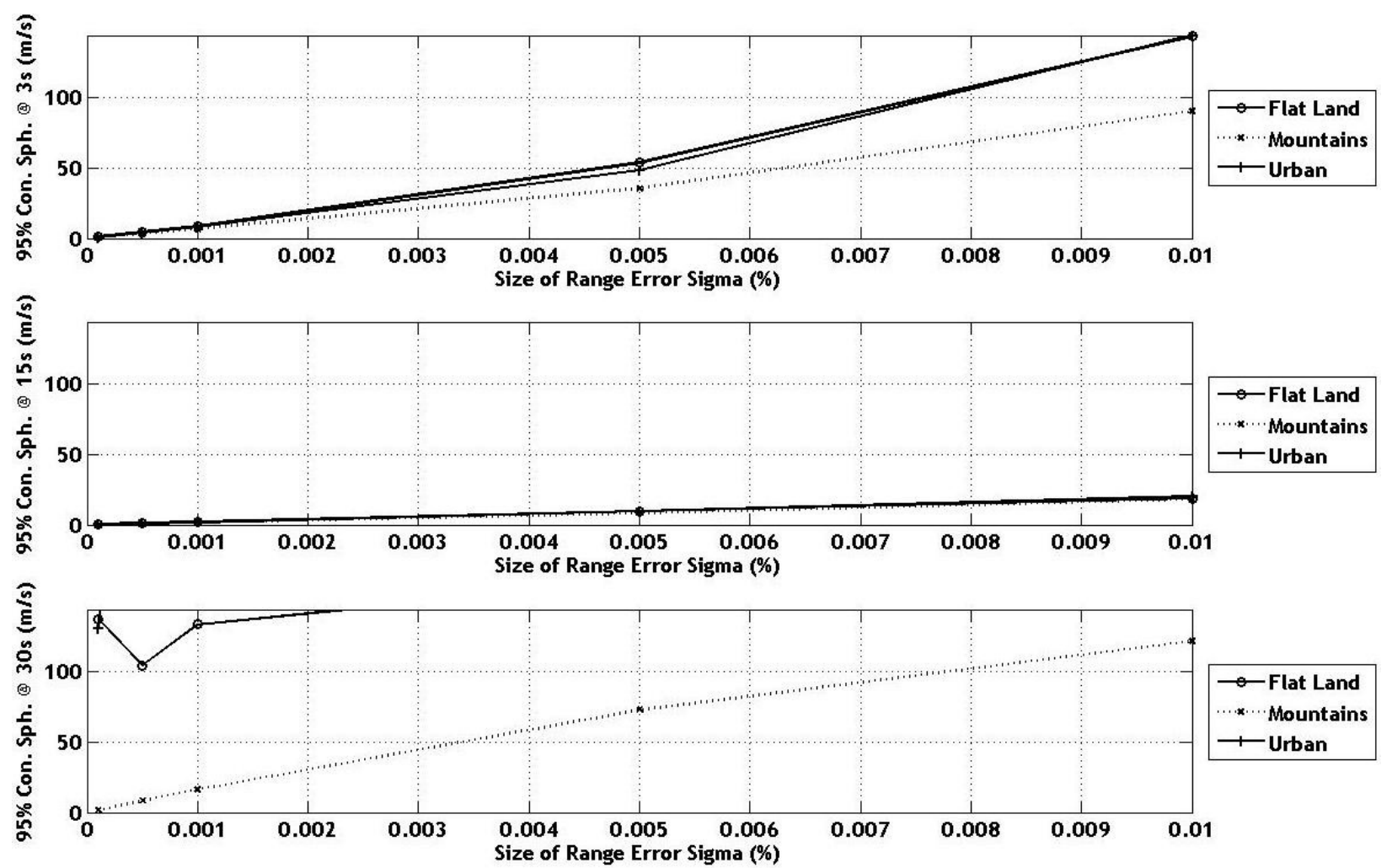

Figure 23. 95\% Confidence Velocity Spheres vs. Size of Error Level for 3s, 15s and 30s

As of now, the parametric trade studies have focused mainly on studying the effects of signal density and error in the signals, but not on errors of the signal source positions themselves. In order to accomplish a full analysis on how these errors affect the accuracy, the signal source positions were randomly perturbed from their actual location by $0.03,0.15,0.3,1.5$ or $3 \mathrm{~m}$. Similar to the previous parametric trade study on error levels, their tends to be an overall upward trend correlating to increasing estimation errors due to increasing signal source position errors, which is displayed on Figure 24. However, not much can be said as to the form of the trade off, because at some points it appears to be linear at others exponential. The same is true for velocity estimations in Figure 25. 

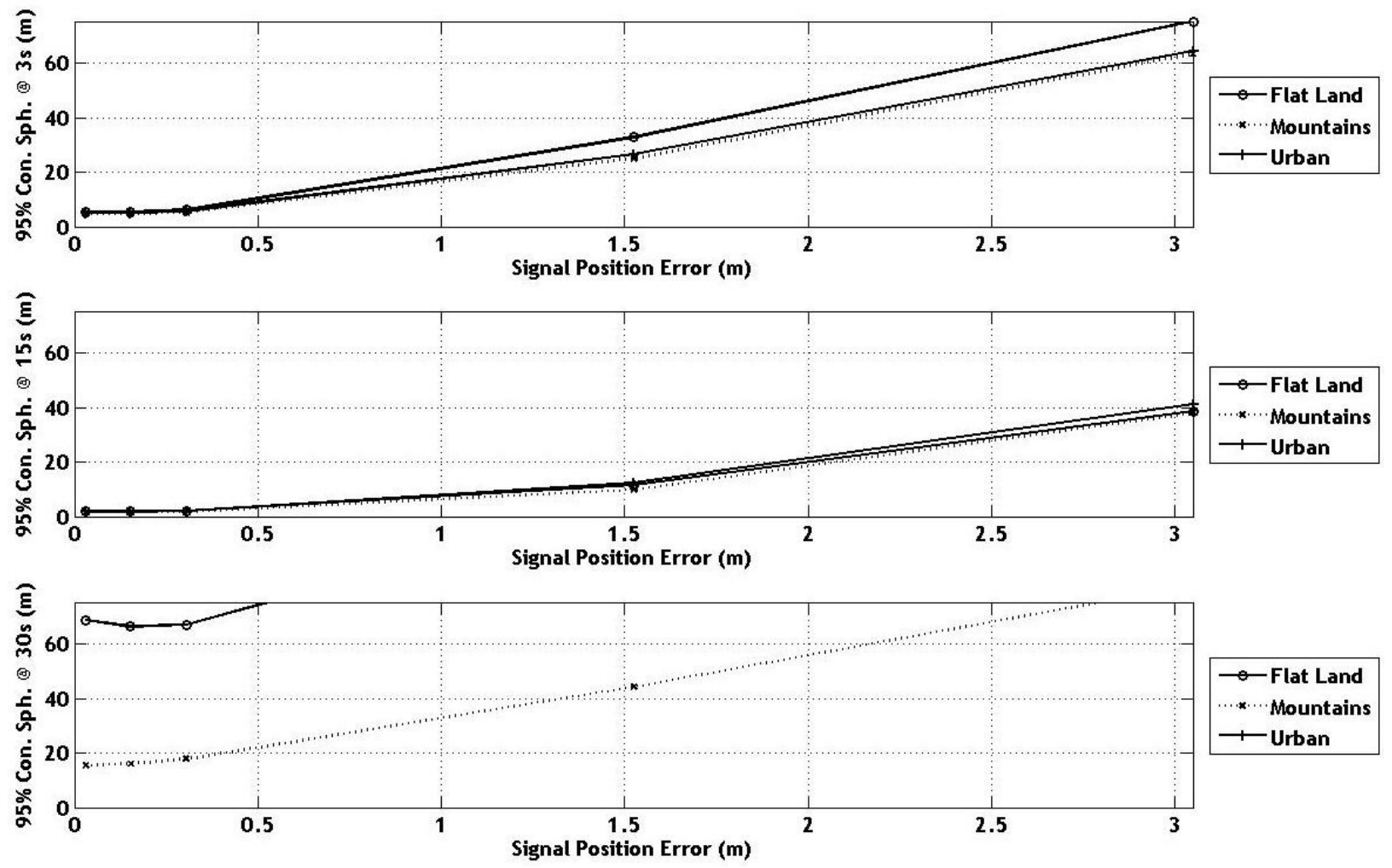

Figure 24. 95\% Confidence Position Spheres vs. Error in Signal Source Position for 3s, 15s and 30s 

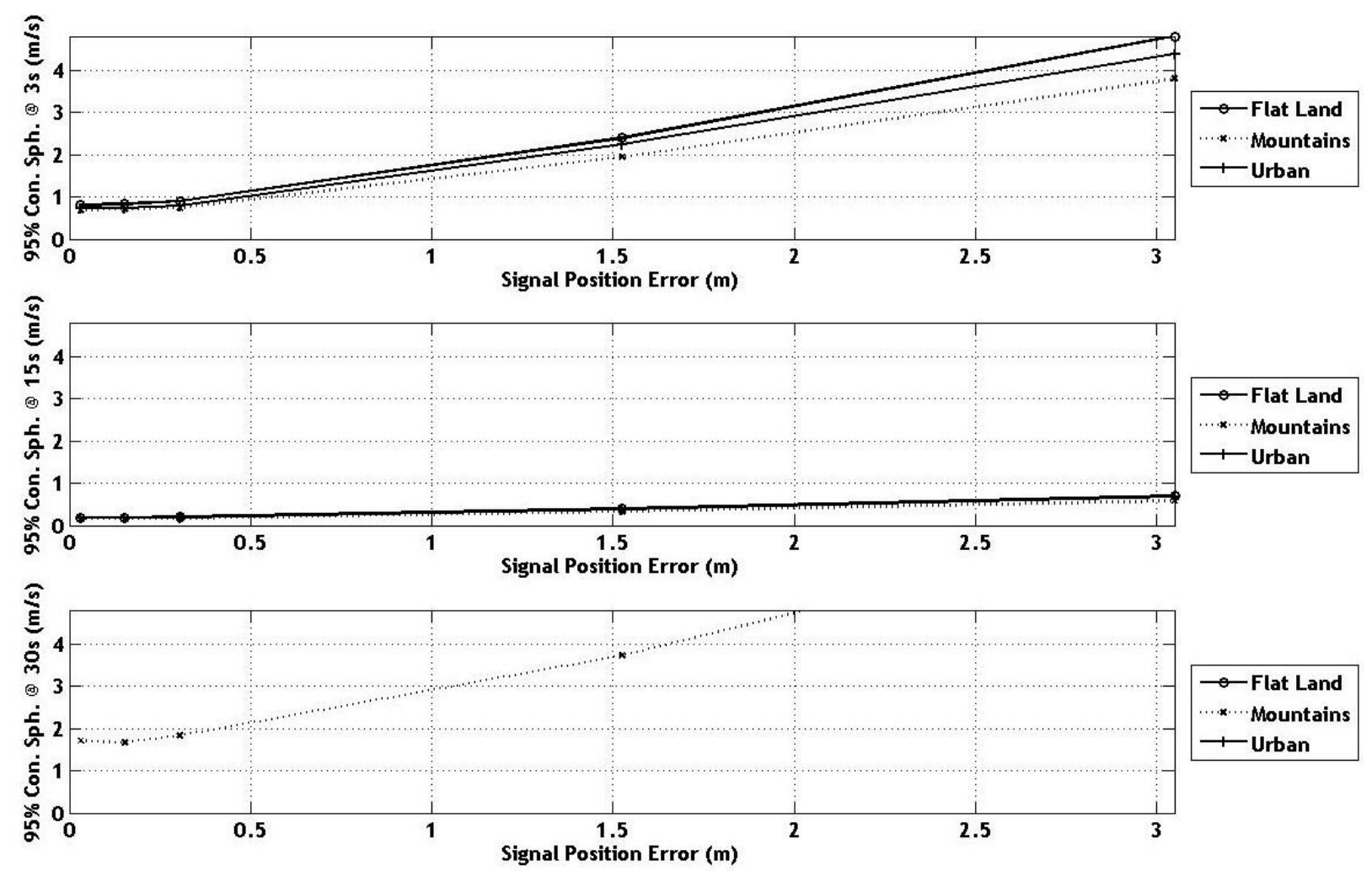

Figure 25. 95\% Confidence Velocity Spheres vs. Error in Signal Source Position for 3s, 15s and 30s

Based on these parametric trade studies and experimental results from journal articles, a set of mission profiles were selected in order to demonstrate a best, medium and worst case scenario. Each scenario was performed on all 3 elevation data sets, and the signal sources were centered around the target in a $4.6 \mathrm{~km}$ square. The values selected for the best case were, 200 signal sources with error levels: $\sigma=0.0001 \& b=0.3 \mathrm{~m}$, and signal source misposition of $0.3 \mathrm{~m}$. For the medium scenario, 100 signal sources were used with error levels of $\sigma=0.001 \& b=1.5$ $\mathrm{m}$, and signal source misposition of $1.5 \mathrm{~m}$. Worst case consisted of only 25 signal sources with $\sigma$ $=0.01 \& b=3 \mathrm{~m}$ error levels, and a signal source misposition of $3 \mathrm{~m}$. The results for $\mathrm{t}=3 \mathrm{~s}, 15 \mathrm{~s}$ and 30s is shown in Table 3. As expected, the best case scenario significantly outperformed the 
two additional scenarios. With respect to elevation datasets, the mountainous landscape outperformed the remaining landscapes, mainly due to the fact that it allowed the altitude of signal sources to vary the most. Taking the best case scenario metrics into consideration, the best case scenario is capable of producing results similar to GPS driven navigation solutions.

Table 3. 95\% Confidence Spheres vs. Scenarios for 3s, 15s and 30s

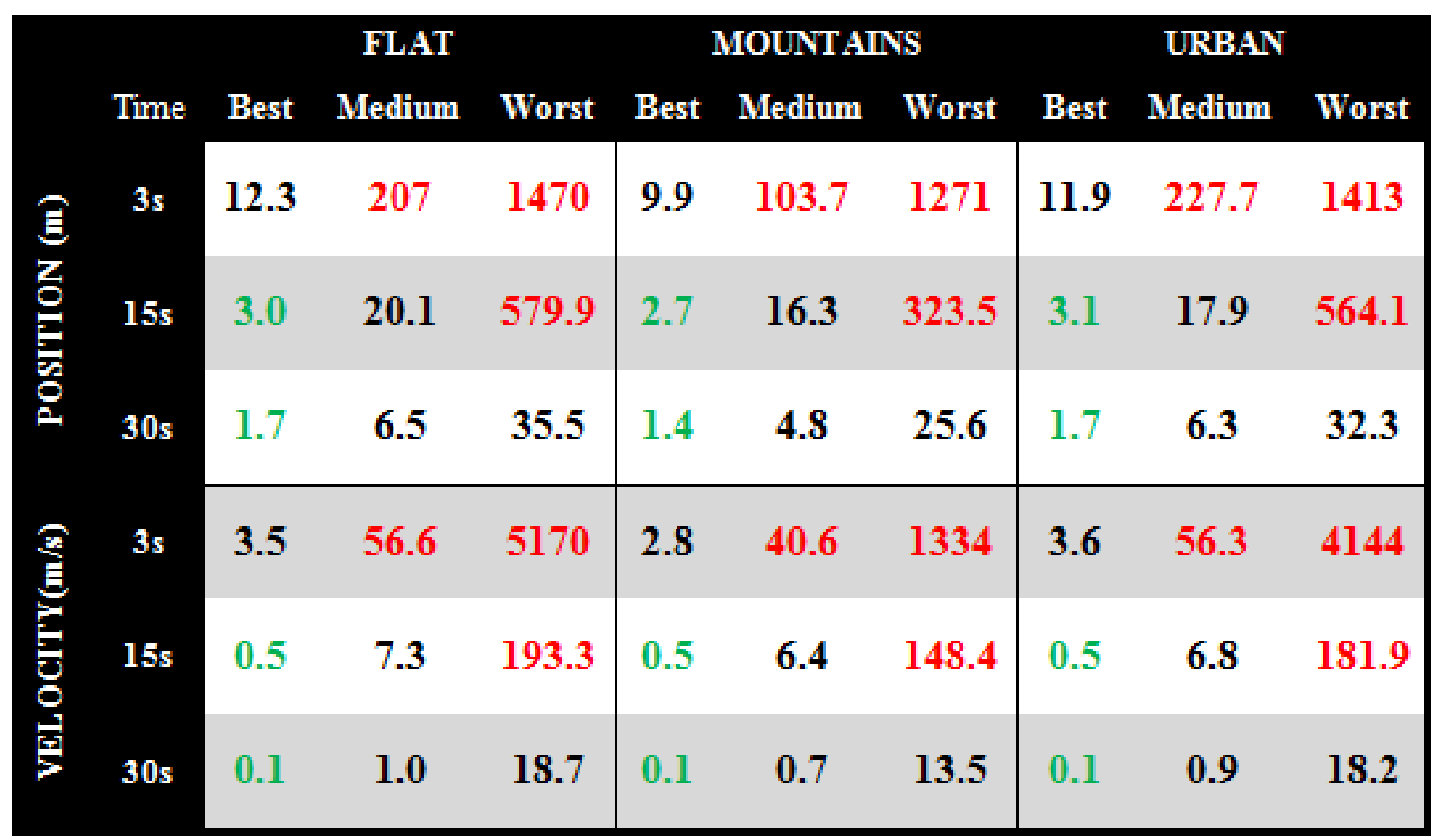




\section{CHAPTER 6 - SUMMARY}

A general method was created to evaluate the ability of ground based range and range rate information to be used by a projectile to construct a navigation solution. Systematic studies were performed to better understand the merits and demerits of this type of system to create a useful navigation solution. Based on these studies, highly accurate navigation solutions stem from relatively large variations in altitude among the signal sources, typically on the order of tens of meters. Also, this variation should also be applied to $\mathrm{X}$ and $\mathrm{Y}$ positioning of signal sources, but with a concentration around where the navigation solution needs to be the most accurate, which is typically near the target. The amount of RF ground based signals do play an important role in accuracy, however there is a point of diminishing return. By also choosing specific signals and additional metrics that reduce the overall error in certain parameters, the accuracy of navigation will be improved significantly. Based on these parametric studies, a cluster of properly placed, currently available, ground based RF signals are able to produce navigation solutions equal in accuracy to GPS and can be used as a practical navigation solution when GPS is not available. 


\section{REFERENCES}

1) Parsch, Andreas. "Texas Instruments Paveway I \& Pave Storm." Directory of U.S. Military Rockets and Missiles. Texas Instruments, 2006. Web. <http://www.designationsystems.net/dusrm/app5/paveway-1.html>.

2) Parsch, Andreas. "Boeing (McDonnell Douglas) JDAM." Directory of U.S. Military Rockets and Missiles. Texas Instruments, 2006. Web. <http://www.designationsystems.net/dusrm/app5/jdam.html>.

3) Abbott, Anthony. "Antijamming and GPS for Critical Military Applications." Crosslink. Aerospace Corporation, Summer 2002. Web. $<$ http://www.aero.org/publications/crosslink/summer2002/06.html>.

4) Defense Update. "GPS Anti-Jam Receiver (AGR)." Defense Update. 26 Jan. 2005. Web. $<$ http://defense-update.com/products/g/gps-ajr.htm>.

5) Williams, Paul, Sally Basker, and Nick Ward. "ENavigation and the Case for eLORAN." Journal of Navigation 61 (2008): 473-84.

6) Savarese, Chris, Jan Rabaey, and Jan Beutel. "LOCATIONING IN DISTRIBUTED ADHOC WIRELESS SENSOR NETWORKS." 2001 IEEE International Conference on Acoustics, Speech, and Signal Processing 4 (2001): 2037-2040.

7) Yang, Po, Wenyan Wu, Mansour Moniri, and Claude C. Chibelushi. "SLAM Algorithm for 2D Object Trajectory Tracking based on RFID Passive Tags." IEEE International Conference on RFID (2008): 165-72.

8) Bekkali, Abdelmoula, Horacio Sanson, and Mitsuji Matsumoto. "RFID Indoor Positioning Based on Probabilistic RFID Map and Kalman Filtering." Third IEEE International Conference on Wireless and Mobile Computing, Networking and Communications (2007): 21-21.

9) Enhanced Loran (eLoran). Issue brief. 0.1th ed. International Loran Association, 2007.

10) The Case for eLORAN. Tech. General Lighthouse Authorities of the United Kingdom and Ireland, 2006. 
11) Offermans, Gerard, Arthur Helwig, Paul Williams, and Wouter Pelgrum. "Differential eLoran trials in Harwich harbour." 35th ILA Convention. Groton. 23 Oct. 2006. Lecture.

12) Trilateration. Wikipedia. Web. <http://en.wikipedia.org/wiki/Trilateration>.

13) McEllroy, Jonathan. "Navigation Using Signals of Opportunity in the AM Transmission Band." Thesis. Air Force Institute of Technology, 2006.

14) Hall, Timothy. "Radiolocation Using AM Broadcast Signals." Thesis. Massachusetts Institute of Technology, 2002. 